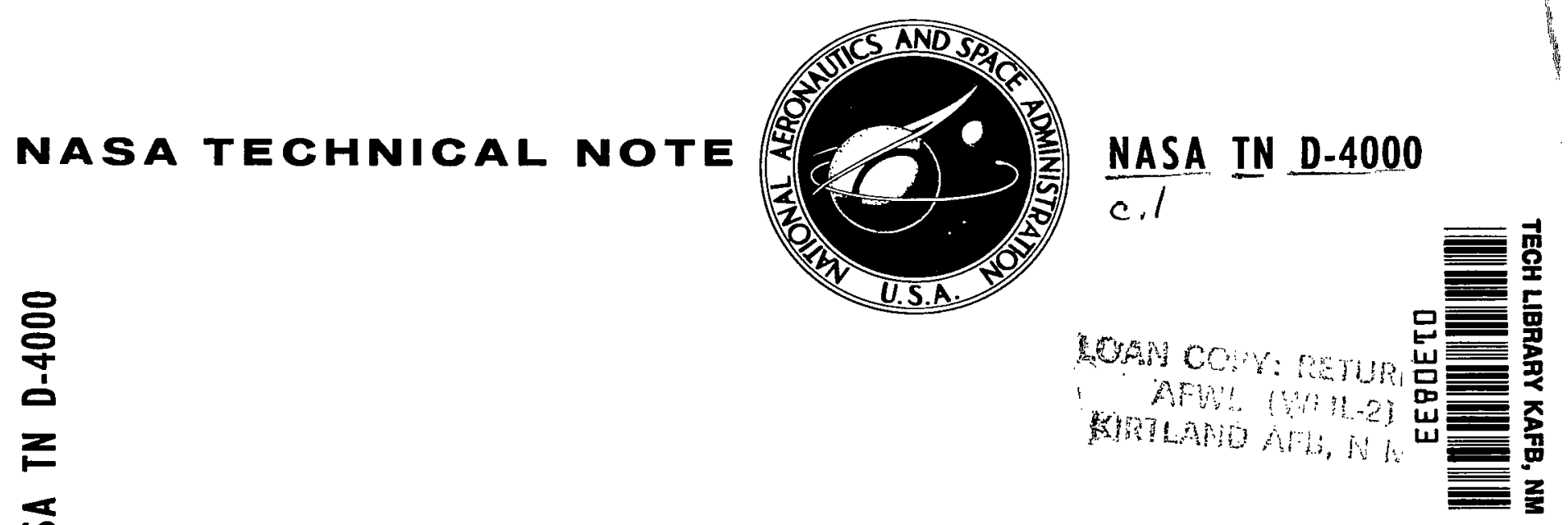

\title{
GENERALIZED POTENTIALS \\ FOR INELASTIC SCATTERING
}

by Howard C. Volkin

Lewis Research Center

Cleveland, Obio

NATIONAL AERONAUTICS AND SPACE ADMINISTRATION - WASHINGTON, D. C.i MAY 1967 
NASA TN D-4000

\title{
GENERALIZED POTENTIALS FOR INELASTIC SCATTERING
}

\author{
By Howard C. Volkin \\ Lewis Research Center \\ Cleveland, Ohio
}

\section{NATIONAL AERONAUTICS AND SPACE ADMINISTRATION}

For sale by the Clearinghouse for Federal Scientific and Technical Information Springfield, Virginia 22151 - CFSTI price $\$ 3.00$ 


\title{
GENERALIZED POTENTIAL FOR INELASTIC SCATTERING*
}

\author{
by Howard C. Volkin
}

\author{
Lewis Research Center
}

\section{SUMMARY}

The generalized potential that gives the scattering into a selected group of inelastic channels along with the elastic scattering is derived by means of the projection operator for the set of channels. A general class of projection operators that selects all open channels is developed in terms of the previous projection operator. The resonance part of the generalized potential can then be obtained by means of Feshbach's unified theory of nuclear reactions. There is no limitation on the types of reactions that can occur. Examples of projectors belonging to the class are given for the case of two-channel reactions. An example which is discussed in some detail is the pickup process. After the resonance contributions have been isolated, the transition amplitudes can be energy averaged. The result is used to obtain the generalized optical potential. The properties of the potentials that follow from the equations which determine them are discussed. A short review is given of some elements of Feshbach's reaction theory that are required.

\section{INTRODUCTION}

The complex potential model has proved to be very useful for representing the elastic scattering of particles by complicated target systems in a wide variety of situations. More recently, with the continuing improvement in computer technology, the use of generalized potentials has become practical and has given equally impressive results. Many derivations of the exact single-channel potentials have been given (refs. 1 to 7). They lead to formal expressions for a potential which gives precisely the same elastic scattering as that determined by the many-body Schrödinger equation from which the probelm starts.

Multichannel or generalized potentials have been derived by Feshbach (refs. 1 and 2) and Mittleman and Pu (refs. 8 and 9). None of these treatments, however, combine all

\footnotetext{
* To be published in the Physial Review.
} 
the following conditions:

(1) There is no limitation on the types of reactions that can occur.

(2) Resonance contributions to the potential are isolated and shown explicitly.

(3) The potential obtained describes the elastic and any selected part of the inelastic scattering.

In medium-energy nuclear reactions, however, all these conditions must be met in order to relate directly an exact potential to a phenomenological potential of the kind employed in a coupled-channel calculation for the elastic and inelastic scattering. The primary purpose of this report is to show how the generalized potentials can be derived when all three of the preceding conditions are fulfilled. In the course of treating this problem, several useful properties of channel projection operators are developed. Furthermore, a rigorous procedure for including resonances in coupled-channel calculations is exhibited as part of the overall development.

The term generalized potential refers to a simplified Hamiltonian which will yield a scattering solution with the same asymptotic behavior in a certain group of channels as that which the scattering state of the full Hamiltonian, with the corresponding boundary conditions, has in these channels. The term generalized optical potential is reserved to designate a complex potential that produces, at an energy $\mathbf{E}$, transition amplitudes that are equal to the energy-averaged transition amplitudes of the system when the average is taken over a suitable energy interval about the value $\mathrm{E}$. (Symbols are defined in appendix A.) The counterpart employed in applications is a phenomenological potential model that possesses internal degrees of freedom corresponding to a particular kind of target excitation. The model is used to describe the inelastic scattering for a selected set of target excitations of the type represented by the particular model, as well as the elastic scattering. The approach leads to a set of coupled equations for the relative motion part of the wave-function components in these channels. Solving the set of equations yields, concurrently, the scattering given by the model in all the included channels.

In direct nuclear reactions, there frequently occur inelastic scattering amplitudes for the excitation of highly collective states of the target nucleus which are almost as large as the elastic amplitude. In such cases, approximations for the inelastic scattering which neglect the coupling between these strongly excited channels are often inaccurate. At the same time, the single-channel optical model may require unusual values for its parameters in order to describe the direct elastic scattering. A set of coupled equations must be used to obtain satisfactory results. As the findings of coupled-channel calculations (refs. 10 to 12) accumulate, it is expected that the empirical properties of generalized optical potentials will become as well established as those of the single-channel optical model and provide information about the nucleus of comparable importance.

In the next section, GENERALIZED COMPLEX POTENTIALS, a generalized potential is derived (in a way that will be useful later) that gives the scattering in a chosen set of 
inelastic channels along with the elastic scattering. This result has been given recently by Mittleman and Pu (refs. 8 and 9 ) and it satisfies conditions (1) and (3). This kind of potential, however, is not well suited to the situation where the scattering exhibits resonance effects because of the formation of quasi-stationary bound states of the total system. In this case, what is usually needed for comparison with experimental results is the optical potential. To obtain the optical potential, it is first necessary to separate explicitly the resonance part of the transition amplitude, which contains the rapid energy dependence, from the slowly varying part. For this purpose the formalism of Feshbach's unified theory of nuclear reactions (refs. 1 and 2) is particularly convenient. Feshbach has obtained expressions for generalized potentials, both the monoenergetic and optical variety, which describe the reactions into all the open channels. In reference 1 , the development is limited to the case where only inelastic reactions occur and thereby satisfies conditions (2) and (3). In reference 2, the treatment is extended to general reactions and so meets the requirements of conditions (1) and (2). In appendix B, some basic elements of Feshbach's reaction theory that are required herein are reviewed.

In this report the potentials which satisfy all three of the preceding conditions are derived. The key step in the development is to relate the projection operator $P$, which selects all the open channels, and the projection operators employed in the section GENERA LIZED COMPLEX POTENTIALS, which select the inelastic channels. This is accomplished in the section SUBSPACES AND THEIR PROJECTORS. First, the properties of the $\mathbf{P}$ projectors are described; a form for $\mathrm{P}$ is chosen that has the desired relation; and then the general expressions are applied to reactions that involve only two types of channels. By way of illustration, the results are discussed for the case of pickup or stripping processes in appendix $\mathrm{C}$. The generalized potential, which describes the scattering in the selected inelastic channels, is obtained in a form that clearly exhibits the resonance contribution. Appropriate energy averaging then leads to the corresponding generalized optical potential. Finally, some of the properties of these potentials that follow from the equations which determine them are discussed.

\section{GENERALIZED COMPLEX POTENTIALS}

The collision of particles that are themselves bound aggregates of elementary particles is now considered. A reaction channel is identified by the indices $\alpha$ and $\mathrm{n}$ which designate, respectively, the distribution of fundamental particles into bound fragments and the internal states of these fragments. For simplicity, all particles are treated as distinguishable. Then, for a given set of bound fragments $\alpha$, the Hamiltonian $\mathscr{H}$ can be separated into a noninteracting Hamiltonian $\mathrm{K}_{\alpha}$ and an interaction $\mathbf{V}_{\alpha}$; 


$$
\mathscr{H}=\mathrm{K}_{\alpha}+\mathrm{V}_{\alpha}
$$

The operator $\mathrm{K}_{\alpha}$ contains the kinetic energy of each elementary particle and its interaction with every other particle of the bound fragment in which it is found in the set $\alpha$ and may conveniently be expressed as

$$
\mathbf{K}_{\alpha}=\mathbf{T}_{\alpha}+\mathscr{x}_{\alpha}
$$

where each term on the right consists of a sum over all the $\alpha$ fragments, $\mathrm{T}_{\alpha}$ the sum of the center-of-mass kinetic energies, and $\mathscr{x}_{\alpha}$ the sum of the internal Hamiltonians. An eigenstate $\varphi_{\alpha \mathrm{n}}$ of $\mathscr{x}_{\alpha}$ consists of the product of the state of motion of each fragment relative to its fixed mass center. An eigenstate $\mathrm{u}_{\alpha \overrightarrow{\mathrm{k}}}$ of $\mathrm{T}_{\alpha}$ can be taken to be a product of plane waves corresponding to the free motion of the mass centers, where $\overrightarrow{\mathrm{k}}$ is used to specify the momenta of the fragments. Noninteracting states, which are eigenstates of $\mathrm{K}_{\alpha}$, can be written as

$$
\varphi_{\alpha \mathrm{n} \vec{k}}=\mathrm{u}_{\alpha \overrightarrow{\mathrm{k}}} \varphi_{\alpha \mathrm{n}}
$$

The indices $\alpha$ o will be reserved for the incident channel. Of course, incident channels consisting of two particles are of primary interest, but the formalism appears the same in the more general case. The continuum eigenstate of $\mathscr{H}$, that describes the reaction, is given by the formal expression

$$
\Psi_{\alpha \mathrm{ok}}^{(+)}=\varphi_{\alpha \mathrm{ok}}+\frac{1}{\mathrm{E}-\mathscr{H}+\mathrm{i} \epsilon} \mathrm{V}_{\alpha} \varphi_{\alpha \mathrm{ok}}
$$

where the limit $\epsilon \rightarrow 0^{+}$is understood on the right. The plus sign on the term if corresponds to the boundary condition that only outgoing waves are present at asymptotic separations of the fragments in the exit channels $\beta \mathrm{m} \neq \alpha$ o. The center-of-mass reference system with the center of mass at the origin is the most useful reference system for our purposes. For notational convenience, the abbreviated form

$$
\Psi \equiv \Psi_{\alpha \mathrm{ok}}^{(+)}
$$

is used for the desired solution (eq. (4)) of the Schrödinger equation

$$
(\mathrm{E}-\mathscr{H}) \Psi=0
$$


In addition to the explicit form of equation (4), the Lippman-Schwinger integral equation is satisfied by $\Psi$ :

$$
\Psi=\varphi_{\alpha \mathrm{ok}}+\frac{1}{\mathbf{E}-\mathbf{K}_{\alpha}+\mathrm{i} \epsilon} \mathrm{V}_{\alpha} \Psi
$$

Consider now the subspace $\mathscr{V}_{\alpha \mathrm{m}}$ of the total Hilbert space spanned by all states of the form $X \varphi_{\alpha \mathrm{m}}$, where $\chi$ is any state of motion for the mass centers of the particles $\alpha$. Because any two $\varphi_{\alpha \mathrm{m}}$ with different values of $\mathrm{m}$ are orthogonal on the scalar product over the internal variables of the particles, the $\mathscr{V}_{\alpha \mathrm{m}}$ constitute a set of subspaces which which are orthogonal on the full scalar product of the space. The projection operator $\Pi_{\alpha \mathrm{m}}$ into the subspace $\mathscr{V}_{\alpha \mathrm{m}}$ can be written as the outer product

$$
\Pi_{\alpha \mathrm{m}}=\left|\varphi_{\alpha \mathrm{m}}\right\rangle\left\langle\varphi_{\alpha \mathrm{m}}\right|
$$

where it is understood that $\Pi_{\alpha \mathrm{m}}$ acts as a multiple of the unit operator on a state of motion of the mass centers. Then the set of projectors has the following properties:

$$
\begin{aligned}
& \Pi_{\alpha \mathrm{m}} \Pi_{\alpha \mathrm{m}}{ }^{\prime}=\delta_{\mathrm{mm}}{ } \Pi_{\alpha \mathrm{m}} \\
& \Pi_{\alpha \mathrm{m}}^{\dagger}=\Pi_{\alpha \mathrm{m}} \\
& \Pi_{\alpha \mathrm{m}} \varphi_{\alpha \mathrm{m}} \overrightarrow{\mathrm{k}}=\delta_{\mathrm{mm}} \varphi_{\alpha \mathrm{m}} \overrightarrow{\mathrm{k}}
\end{aligned}
$$

and

$$
\left[\Pi_{\alpha \mathbf{m}}, \mathbf{K}_{\alpha}\right]=0
$$

The wave function $\Psi$ can be expanded in terms of the complete orthonormal set of eigenstates of $\mathscr{x}_{\alpha}$

$$
\Psi=\sum_{\mathrm{m}} \mathrm{f}_{\alpha \mathrm{m}} \varphi_{\alpha \mathrm{m}}
$$

where the coefficients $f_{\alpha \mathrm{m}}$ are functions of the center-of-mass positions of the particles in the set $\alpha$. The sum runs over the complete set of eigenstates $\varphi_{\alpha \mathrm{m}}$ including the 
states in the continuum spectra of $\mathscr{x}_{\alpha}$. It then follows directly that

$$
\Pi_{\alpha \mathrm{m}} \Psi=\mathbf{f}_{\alpha \mathrm{m}} \varphi \alpha \mathrm{m}
$$

and that all the information about the asymptotic behavior of $\Psi$ in the channel $\alpha \mathrm{m}$ is contained in the projection $\Pi_{\alpha \mathrm{m}} \Psi$.

A set I of $\alpha$ channels, which includes the incident channel $\alpha$ o but otherwise consists of an arbitrary selection of $\alpha$ channels, is now considered. The operator

$$
\Pi_{I}=\sum_{\mathrm{m} \subset \mathrm{I}} \Pi_{\alpha \mathrm{m}}
$$

projects into the vector or direct sum $\mathscr{V}_{I}$ of the selected orthogonal spaces:

$$
\mathscr{V}_{\mathrm{I}}=\mathscr{V}_{\alpha \mathrm{o}} \oplus \mathscr{V}_{\alpha \mathrm{i}}^{\oplus} \ldots \oplus \mathscr{V}_{\alpha \mathrm{j}} \quad \mathrm{o}, \mathrm{i}, \ldots, \mathrm{jcI}
$$

The projector into the entire subspace orthogonal to $\mathscr{V}_{I}$ is

$$
\Theta_{I}=1-\Pi_{I}, \Theta_{I} \Pi_{I}=0
$$

The projections of $\Psi$ are

$$
\Psi_{\mathrm{I}}=\Pi_{\mathrm{I}} \Psi=\sum_{\mathrm{m} \subset \mathrm{I}} \mathrm{f}_{\alpha \mathrm{m}}{ }^{\varphi} \alpha \mathrm{m}
$$

and

$$
\Psi^{\prime}=\Theta_{I^{\Psi}}
$$

Operating on equation (5) with $\Pi_{I}$ and $\Theta_{I}$ gives the pair of coupled equations:

$$
\left(\mathrm{E}-\Pi_{\mathrm{I}} \mathrm{H}^{\left.\mathscr{} \Pi_{\mathrm{I}}\right) \Psi_{\mathrm{I}}}=\Pi_{\mathrm{I}} \mathscr{H} \Theta_{\mathrm{I}} \Psi^{\prime}\right.
$$

and

$$
\left(\mathrm{E}-\Theta_{\mathrm{I}} \mathscr{H} \Theta_{\mathrm{I}}\right) \Psi^{\prime}=\Theta_{\mathrm{I}} \mathscr{H} \Pi_{\mathrm{I}}{ }_{\mathrm{I}}
$$


From the commutation relation (eq. (7d)) it is seen that

$$
\Pi_{I^{*}} \Pi_{I}=K_{\alpha} \Pi_{I}+\Pi_{I} v_{\alpha} \Pi_{I}, \Pi_{I} \mathscr{H} \Theta_{I}=\Pi_{I} v_{\alpha} \Theta_{I} \text {, etc. }
$$

Equation (12b) can be solved as follows:

$$
\Psi^{\prime}=\frac{1}{\mathbf{E}-\mathbf{K}_{\alpha}+\mathrm{i} \epsilon} \Theta_{\mathbf{I}} \mathrm{V}_{\alpha} \Psi
$$

This solution is just the result obtained by operating on equation (6) with $\Theta_{\mathbf{I}}$. The plus sign on the i $\epsilon$ term ensures that, at large separations, only outgoing waves appear in the open inelastic channels not contained in $I$ and in the contribution of $\Psi^{\prime}$ in the rearrangement channels. The imagninary term can be omitted if $I$ contains all the open channels. The vector $\mathrm{V}_{\alpha} \Psi$ satisfies the equation

$$
\mathrm{V}_{\alpha} \Psi=\mathrm{V}_{\alpha} \Psi_{\mathbf{I}}+\mathrm{V}_{\alpha} \frac{1}{\mathbf{E}-\mathbf{K}_{\alpha}+\mathbf{i} \epsilon} \Theta_{\mathbf{I}} \mathrm{V}_{\alpha} \Psi
$$

If an operator $U_{I}^{(+)}$is defined by the equation

$$
\mathrm{U}_{\mathrm{I}}^{(+)}=\mathrm{v}_{\alpha} \Pi_{\mathrm{I}}+\mathrm{V}_{\alpha} \frac{1}{\mathrm{E}-\mathrm{K}_{\alpha}+\mathrm{i} \epsilon} \Theta_{\mathrm{I}} \mathrm{U}_{\mathrm{I}}^{(+)} \Pi_{\mathrm{I}}
$$

it is clear that

$$
\mathrm{U}_{\mathrm{I}}^{(+)} \Psi=\mathrm{U}_{\mathbf{I}}^{(+)} \Psi_{\mathbf{I}}
$$

satisfies the same equation (eq. (14)) as does $V_{\alpha} \Psi$ and, hence, that

$$
\mathrm{v}_{\alpha} \Psi=\mathrm{U}_{\mathrm{I}}^{(+)} \Psi_{\mathrm{I}}
$$

The propagator in equation (14),

$$
\left(E-K_{\alpha}+i \epsilon\right)^{-1} \Theta_{I}=\Theta_{I}\left(E-K_{\alpha}+i \epsilon\right)^{-1} \Theta_{I}
$$

can be rewritten as 


$$
\begin{aligned}
\left(E-K_{\alpha}+\mathrm{i} \epsilon\right)^{-1} \Theta_{I} & =\Theta_{I}\left(E-K_{\alpha}-\Theta_{I} V_{\alpha}+i \epsilon\right)^{-1} \Theta_{I}\left[1-v_{\alpha}\left(E-K_{\alpha}+\mathbf{i} \epsilon\right)^{-1} \Theta_{I}\right] \\
& =\left(E-\Theta_{I} \not \mathscr{t}+\mathbf{i} \epsilon\right)^{-1} \Theta_{I}\left[1-v_{\alpha}\left(E-K_{\alpha}+\mathbf{i} \epsilon\right)^{-1} \Theta_{I}\right]
\end{aligned}
$$

Inserting the resultant identity into equation (15) gives an explicit formal solution of the equation, namely,

$$
\mathrm{U}_{\mathrm{I}}^{(+)}=\mathrm{V}_{\alpha} \Pi_{\mathrm{I}}+\mathrm{V}_{\alpha} \Theta_{\mathrm{I}} \frac{1}{\mathrm{E}-\Theta_{\mathrm{I}} \mathscr{H}+\mathrm{i} \epsilon} \Theta_{\mathrm{I}} \mathrm{V}_{\alpha} \Pi_{\mathrm{I}}
$$

This last result could have been obtained directly by noting that the solution of equation (12b) can also be written as

$$
\Psi^{\prime}=\left(E-\Theta_{I} \mathscr{H} \Theta_{I}+i \epsilon\right)^{-1} \Theta_{I} V_{\alpha} \Psi_{I}
$$

The generalized potential is defined as

$$
\mathrm{V}_{\mathrm{I}}^{(+)}=\Pi_{I} \mathrm{U}_{\mathbf{I}}^{(+)}
$$

and has the property that

$$
\Pi_{I} V_{\alpha} \Psi=V_{I}^{(+)} \Psi_{I}
$$

Equation (12a) can then be written in the form

$$
\left(E-K_{\alpha}-V_{I}^{(+)}\right) \Psi_{I}=0
$$

which shows that the potential $V_{\mathrm{I}}^{(+)}$will give the true scattering in the channels $I$. The generalized potential of equation (17) has recently been applied by $\mathrm{Pu}$ (ref. 13) to the scattering of electrons by atomic hydrogen. 


\section{SUBSPACES AND THEIR PROJECTORS}

\section{Properties of Projectors That Select All Open Channels}

The generalized potential given by equation (17) is not convenient when the scattering shows resonance behavior. The potential will then have a rapid energy variation corresponding to poles in its energy dependence. The origin of this effect lies in the possibility of the system forming compound states with an appreciable lifetime for decay into the exit channels. In order to isolate the contribution to a generalized potential arising from this aspect of system behavior, one of the fundamental theories of reactions must be employed, which will provide a formalism for treating resonances. The theory of nuclear reactions due to Feshbach is particularly appropriate, because it supplies a unified description of direct and resonance reactions.

Reaction channels that are energetically allowed are called open channels, and all others are closed channels. In the open channel $\beta \mathrm{m}$, the wave function $\Psi$ at large separations of the $\beta$ fragments assumes the form

$$
\Psi \overrightarrow{\beta \mathrm{m}^{\prime}} \mathbf{f}_{\beta \mathrm{m}} \varphi_{\beta \mathrm{m}}
$$

A projection $\mathrm{P}$ which selects the open channels is a projection operator for which $\mathrm{P \Psi}$ has the same asymptotic form in any of the open channels as $\Psi$ itself. Thus, at large particle separations in the open channel $\beta \mathrm{m}, \mathrm{P} \Psi$ must yield

$$
\mathrm{P} \Psi \overrightarrow{\beta \mathrm{m}} \mathbf{f}_{\beta \mathrm{m}} \varphi_{\beta \mathrm{m}}
$$

The open-channel subspace into which $\mathrm{P}$ projects will be called $\mathscr{P}$. Since only the as-

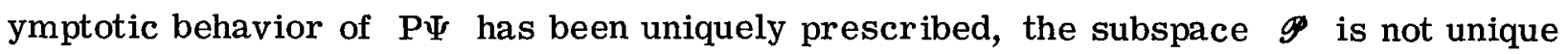
and, hence, neither is the projector $\mathrm{P}$. The largest subspace $\mathscr{Q}$ orthogonal to $\mathscr{P}$ can be referred to as the closed-channel subspace, and its projector is $Q=1-P$. Since the incident channel and all other open channels are included in $P$, when $\mathbf{Q}$ operates on $\mathbf{a}$ state $\Psi$, it yields a function which in any channel has the asymptotic behavior

$$
\mathrm{Q} \Psi \overrightarrow{\beta \mathrm{m}}^{0} \quad \text { for any } \beta \mathrm{m}
$$

In configuration space, $Q \Psi$ is limited essentially to the regions in which fragments of an open channel are interacting.

The pair of coupled equations obtained by operating on equation (5) in turn with $P$ and $Q$ is 


$$
\begin{aligned}
& \left(\mathrm{E}-\mathscr{H}_{\mathrm{PP}}\right) \mathrm{P} \Psi=\mathscr{H}_{\mathrm{PQ}} \mathrm{Q \Psi} \\
& \left(\mathrm{E}-\mathscr{H}_{\mathrm{QQ}}\right) \mathrm{Q \Psi}=\mathscr{H}_{\mathrm{QP}} \mathrm{P} \Psi
\end{aligned}
$$

In view of the boundary condition (eq. (21)) that $Q \Psi$ satisfies, the formal solution of equation (22b) can be written as

$$
\mathrm{Q} \Psi=\frac{1}{\mathrm{E}-\mathscr{H}_{\mathrm{QQ}}} \mathscr{H}_{\mathrm{QP}} \mathrm{P} \Psi
$$

When equation (23) is substituted into equation (22a), a Schrödinger equation for $P \Psi$ is obtained

$$
(E-H) P \Psi=0
$$

with the effective Hamiltonian $\mathrm{H}$ given by

$$
\mathrm{H}=\mathscr{H}_{\mathrm{PP}}+\mathscr{H}_{\mathrm{PQ}} \frac{1}{\mathrm{E}-\mathscr{H}_{\mathrm{QQ}}} \mathscr{H}_{\mathrm{QP}}
$$

The transition amplitudes obtained from $P \Psi$ are exactly those given by the full state vector $\Psi$; thus, resonance effects occurring in the reactions are accompanied by rapid energy variations in the effective Hamiltonian $H$ that governs the behavior of $P \Psi$. It is, in fact, the second term on the right side of equation (25) from which the rapidly varying part of $\mathrm{H}$ arises. The compound states of the total system are described approximately by the eigenstates $\varphi_{\nu}$ of $\mathscr{H}_{\mathrm{QQ}}$ belonging to the discrete spectrum

$$
\left(\mathscr{E}_{\nu}-\mathscr{H}_{\mathrm{QQ}}\right) \varphi_{\nu}=0
$$

These eigenstates are true bound states and, hence, have an infinite lifetime. In addition, there is the continuous spectrum of eigenvalues that begins at a minimum value equal to the smallest threshold energy for opening any of the channels which are closed at the energy $\mathbf{E}$ and which are, thus, not in the set of open channels selected by $\mathrm{P}$. Solutions of the homogeneous form of equation (22b) are $\delta\left(E-\mathscr{E}_{\nu}\right) \varphi_{\nu}$, but any admixture of these functions to the solution given by equation (23) is ruled out by the physical requirement that 


$$
\left(\varphi_{\nu}|\mathrm{Q} \Psi\rangle=\left(\mathrm{E}-\mathscr{E}_{\nu}\right)^{-1}\left\langle\varphi_{\nu}\left|\mathscr{H}_{\mathrm{QP}}\right| \mathrm{P} \Psi\right\rangle\right.
$$

be well behaved as $\mathrm{E}-\mathscr{E}_{\nu}$.

The exact compound state is described by $Q \Psi$ and decays into the open channels by virtue of the coupling provided by the term on the right of equation (22b). But, for values of $\mathrm{E}$ in the neighborhood of an isolated resonance, the compound state has a long lifetime and the rapidly varying part of $\mathrm{Q \Psi}$ is proportional to the state $\varphi_{\nu}$ whose eigenvalue $\mathscr{E}_{\nu}$ is close to $\mathrm{E}$. At an energy where resonances overlap, a linear combination of the states $\varphi_{\nu}$, whose eigenvalues lie within the region of overlap, approximates the compound state. These properties are verified by expanding the operator $\left(\mathrm{E}-\mathscr{H}_{\mathrm{QQ}}\right)^{-1}$ in the total set of eigenstates of $\mathscr{H}_{\mathrm{QQ}}$, including those in the continuous spectrum, and by then using the expansion in equation (23). When the same form of the operator is employed in equation (25), $\mathrm{H}$ can be separated at an energy value $\mathrm{E}$, where resonance occurs into two parts, one of which, $\mathrm{H}^{\prime}$, is slowly varying, and the other, $\mathrm{H}_{\mathrm{R}}$, is rapidly varying with energy:

$$
\mathrm{H}=\mathrm{H}^{\prime}+\mathrm{H}_{\mathrm{R}}
$$

The resonant part $H_{R}$ consists of a single term from the expansion when $E$ is near an isolated resonance. When resonances overlap at $\mathrm{E}, \mathrm{H}_{\mathrm{R}}$ consists of a finite sum of terms corresponding to the contributions of the states $\varphi_{\nu}$ whose eigenvalues $\mathscr{E}_{\nu}$ lie in the region of overlap. The complete derivation of equation (27) is given by Feshbach (ref. 2). The main elements of the development are sketched in appendix B.

\section{Appropriate Forms for Projector That Selects All Open Channels}

Consider now an open channel $\alpha \mathrm{m}$. The projection $\Pi_{\alpha \mathrm{m}} \Psi$ of $\Psi$ into the subspace $\mathscr{V}_{\alpha \mathrm{m}}$ certainly approaches $\Psi$ itself asymptotically in channel $\alpha \mathrm{m}$. However, the full projection into $\mathscr{V}_{\alpha \mathrm{m}}$ is not required to obtain the same asymptotic behavior; that is, there exists, in general, a subspace $\mathscr{V}_{\alpha \mathrm{m}}^{(\alpha)}$ of $\mathscr{V}_{\alpha \mathrm{m}}$ such that the projection of $\Psi$ into this subspace still yields the correct asymptotic result in channel $\alpha \mathrm{m}$. Let $\mathscr{V}_{\alpha \mathrm{m}}^{(\beta, \mathrm{C})}$ designate the largest subspace of $\mathscr{V}_{\alpha \mathrm{m}}$, which is orthogonal to $\mathscr{V}_{\alpha \mathrm{m}}^{(\alpha)}$. Then the projection of $\Psi$ in $\mathscr{V}_{\alpha \mathrm{m}}^{(\beta, C)}$ vanishes asymptotically in channel $\alpha \mathrm{m}$ :

$$
\mathscr{V}_{\alpha \mathrm{m}}=\mathscr{V}_{\alpha \mathrm{m}}^{(\alpha)} \oplus \mathscr{V}_{\alpha \mathrm{m}}^{(\beta, C)}
$$


The corresponding projection operators are

$$
\Pi_{\alpha \mathrm{m}}=\Pi_{\alpha \mathrm{m}}^{(\alpha)}+\Pi_{\alpha \mathrm{m}}^{(\beta, \mathrm{C})}
$$

where

$$
\Pi_{\alpha \mathrm{m}}^{(\alpha)} \Pi_{\alpha \mathrm{m}}^{(\beta, C)}=0
$$

Thus, a decomposition of the subspace $\mathscr{V}_{\alpha \mathrm{m}}$ is introduced which is not necessarily unique but which has the following properties:

$$
\begin{gathered}
\Pi_{\alpha \mathrm{m}}^{(\alpha)} \Psi \underset{\alpha \mathrm{m}}{\longrightarrow} \Psi \\
\Pi_{\alpha \mathrm{m}}^{(\beta, C)} \Psi \underset{\alpha \mathrm{m}}{ } 0
\end{gathered}
$$

and

$$
\Pi_{\alpha \mathrm{m}}^{(\alpha)}(Q \Psi)=0
$$

In order to see that $\mathscr{V}_{\alpha \mathrm{m}}$ is generally a somewhat larger projective space than necessary in which to retain the desired asymptotic information about $\Psi$ in channel $\alpha \mathrm{m}$, other possible requirements can be cited which the subspace $\mathscr{V}_{\alpha \mathrm{m}}$ may have to fulfill. First, consider an open rearrangement channel $\beta \mathrm{n}$ in which some fragments of the set $\alpha$ are bound together and the others are the same, as for example in neutron capture. When the internal state $\varphi_{\beta \mathrm{n}}$ is expanded in the set $\varphi_{\alpha \mathrm{m}}$, clearly, large components for open channel values of $\alpha \mathrm{m}$ can occur. But the contribution of $\mathscr{V}_{\alpha \mathrm{m}}$ that enters into the description of channel $\beta \mathrm{n}$ corresponds to functions that vanish asymptotically in channel $\alpha \mathrm{m}$. The part of $\mathscr{\gamma}_{\alpha \mathrm{m}}$ that yields only functions of this kind cannot enter into the asymptotic behavior of $\Psi$ in channel $\alpha \mathrm{m}$. However, it contributes to that part of $\mathscr{P}$ which plays a role only in the description of rearrangement channels.

A second function that might conceivably be required of $\mathscr{V}_{\alpha \mathrm{m}}$ involves its projection in $\mathscr{Q}$. A restriction that is always implied on the extent of $\mathscr{P}$ is that the closed channel subspace $\mathscr{Q}$ must have enough in it to provide a description of compound states. The compound states in nuclear reactions, however, are expected to have a very small overlap with the relatively low lying states $\varphi_{\alpha \mathrm{m}}$ available to inelastic excitation of the target system. In the case of an incident nucleon, the compound nucleus has an excitation 
above its ground state of the order of $8 \mathrm{MeV}$ plus the kinetic energy of relative motion. The actual complex motion of the compound system can be pictured as reached through a succession of two-body interactions. It has been estimated (ref. 14) that, even at low incident energies, this multiple excitation produces about seven quasi-particles (i. e., eight excited particles and seven holes compared with the initial state of the target). Thus, the portion of $\mathscr{V}_{\alpha \mathrm{m}}$ required for this second purpose is very small but not identically a null space. In both the examples presented, the parts of $\mathscr{V}_{\alpha \mathrm{m}}$ that were utilized meet the criterion established for $\mathscr{V}(\beta, \mathrm{m})$.

To illustrate how the separation of $\mathscr{V}_{\alpha \mathrm{m}}$ might be effected in terms of a set of basis vectors in the subspace, consider a potential function in the mass center coordinates of the $\alpha$ fragments with the same symmetries in these coordinates as $\mathscr{H}$ itself. For nucleon scattering, a shell model potential for the target nucleus would be appropriate. In equation (8), the coefficient $f_{\alpha \mathrm{m}}$ can be expanded in the complete set of eigenfunctions of the potential with suitable outgoing wave boundary condition. For large separations of the mass centers, the discrete bound eigenfunctions $\psi_{\mathrm{i}}$ do not contribute to the expansion. Thus, $\mathscr{\gamma}(\beta, \mathrm{m})$ can be taken to be a space spanned by all or some subset of the basis vectors $\psi_{\mathrm{i}} \varphi_{\alpha \mathrm{m}}$.

The subspaces $\mathscr{V}_{\alpha \mathrm{m}}, \mathscr{V}_{\alpha \mathrm{m}}^{(\alpha)}$, and $\mathscr{V}_{\alpha \mathrm{m}}^{(\beta, \mathrm{C})}$ are orthogonal to all other $\mathscr{V}_{\alpha \mathrm{n}}$, where $\mathrm{n} \neq \mathrm{m}$. Unlike $\mathscr{V}_{\alpha \mathrm{m}}$, however, the orthogonal subspaces $\mathscr{V}_{\alpha \mathrm{m}}^{(\alpha)}$ and $\mathscr{V}_{\alpha \mathrm{m}}^{(\beta, \mathrm{C})}$ are not invariant under the operation of $\mathrm{K}_{\alpha}$, which does not commute with either $\Pi_{\alpha \mathrm{m}}^{(\alpha)}$ or $\Pi_{\alpha \mathrm{m}}^{(\beta, \mathrm{C})}$. Let the set of all open $\alpha$ channels be designated as A. These open channels are enumerated by the indices $\alpha \mathrm{m}$, in order of increasing values of the internal energy $\mathscr{X}_{\alpha}$, when $\mathrm{m}$ runs from 0 to $\mathrm{N}(\alpha)$. Also, let the vector sum of the subspaces $\mathscr{V}_{\alpha \mathrm{m}}$ over the set $\mathrm{A}$ be called $\mathscr{V}_{\mathrm{A}}$, the subspace orthogonal to it be $\mathscr{V}_{\mathrm{A}}^{\prime}$, and the projection operator into $\mathscr{V}_{\mathrm{A}}$ be $\Pi_{\mathrm{A}}$. Then these quantities can be written in the form

$$
\begin{aligned}
& \mathscr{V}_{\mathrm{A}}=\mathscr{V}_{\alpha 1}^{\oplus} \mathscr{V}_{\alpha 2}{ }^{\oplus} \cdot \cdot \cdot \boldsymbol{\oplus}_{\alpha \mathrm{N}}
\end{aligned}
$$

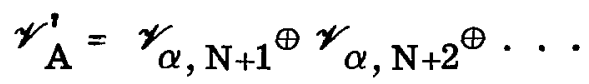

and

$$
\Pi_{\mathrm{A}}=\sum_{\mathrm{m}=0}^{\mathrm{A}} \Pi_{\alpha \mathrm{m}}
$$

and similar expressions for the vector sum $\mathcal{V}_{\mathrm{A}}^{(\alpha)}$ of the subspaces $\mathscr{\gamma}_{\alpha \mathrm{m}}^{(\alpha)}$ over the set $\mathrm{A}$, and its projector $\Pi_{\mathrm{A}}^{(\alpha)}$, etc. can be written. 
Consider the intersection of $\mathscr{P}$ and $\mathscr{V}_{\mathrm{A}}$, which is a linear subspace of both $\mathscr{P}$ and $\mathscr{V}_{\mathrm{A}}$. For an arbitrary projection $\mathrm{P}$, this plane of intersection will be skew to the orthonormal basis for $\mathscr{V}_{\mathrm{A}}$ constructed from the eigenstates of $\mathscr{X}_{\alpha}$ (each vector of the basis set has the form $\xi \varphi_{\alpha \mathrm{m}}$ where $\left.\mathrm{m} \subset \mathrm{A}\right)$. However, there is sufficient flexibility in the selection of a projection into the open channels that in any problem $\mathbf{P}$ can always be chosen so that

$$
\mathscr{P} \cap \mathscr{V}_{\mathrm{A}}=\mathscr{V}_{\mathrm{A}}^{(\alpha)}
$$

This follows because the part of $\mathscr{P}$ required for the description of the asymptotic behavior in the open $\alpha$ channels must also lie in $\mathscr{V}_{\mathrm{A}}$ and, by definition, the subspace $\mathscr{V}_{\mathrm{A}}^{(\alpha)}$ is adequate for this purpose. The subspace $\mathscr{V}_{\mathrm{O}}^{(\beta)}$ in $\mathscr{P}$, which is orthogonal to $\mathscr{V}_{\mathrm{A}}^{(\alpha)}$, is the part of $\mathscr{P}$ which is required only for the description of open rearrangement channels. (The space $\mathscr{V}_{\mathrm{A}}^{(\alpha)}$ may also contribute to rearrangement channels involving bound $\alpha$ fragments.) These subspaces are visualized schematically in figure 1. Thus, $P$ can always be chosen to have the form

$$
\mathbf{P}=\Pi_{\AA}^{(\alpha)}+\Pi_{\mathrm{O}}^{(\beta)}, \Pi_{\AA}^{(\alpha)} \Pi_{\mathrm{O}}^{(\beta)}=0
$$

with the projective space

$$
\mathscr{P}=\mathscr{V}_{\mathrm{A}}^{(\alpha)} \oplus \mathscr{V}_{\mathrm{O}}^{(\beta)}
$$

Such a choice has the advantage that the projective space $\mathscr{V}_{\mathrm{A}}^{(\alpha)}$ of $\Pi_{\mathrm{A}}^{(\alpha)}$, which yields the projection into the open $\alpha$ channels, is spanned by an orthonormal basis whose vectors are all eigenstates of $\mathscr{X}_{\alpha}$. This choice is convenient for separating the elastic and inelastic processes from the remaining reactions. However, none of the spaces in equation (33b) is invariant under $K_{\alpha}$ and none of the projectors in equation (33a) commutes with $\mathbf{K}_{\alpha}$.

From the discussion given previously, the assumption that the compound states have no components in $\mathscr{V}_{\mathrm{A}}$ does not appear to be overly restrictive in nuclear reactions. It follows from this assumption that none of the subspace $\mathscr{V}_{\mathrm{A}}$ is needed in $\mathscr{Q}$ and, hence, that $\mathscr{P}$ can be enlarged to include all of $\mathscr{V}_{\mathrm{A}}$. Then the form

$$
P=\Pi_{A}+\Pi_{O}, \Pi_{A} \Pi_{O}=0
$$

can be chosen with the corresponding relation 


$$
P=\mathscr{V}_{A} \oplus \mathscr{V}_{O}
$$

where $\mathscr{V}_{\mathrm{O}}$ is the subspace in $\mathscr{P}$ which is orthogonal to $\mathscr{V}_{\mathrm{A}}$. Although $\Pi_{\mathrm{O}}$ in equation (34a) does not commute with $\mathrm{K}_{\alpha}, \Pi_{\mathrm{A}}$ (eq. (7d)) does.

\section{Two Kinds of Open Channels}

In order to make more explicit the general arguments given so far, a somewhat simplified reaction where only two kinds of channels are open, the $\alpha$ and the $\beta$ channels, is considered. Let $\mathrm{A}$ and $\mathrm{B}$ designate, respectively, the complete set of open $\alpha$ and open $\beta$ channels, and let $\Pi_{A}$ and $\Pi_{B}$ be the projectors of the sort defined by equation (32) into the respective total subspaces $\mathscr{V}_{A}$ and $\mathscr{V}_{B}$, like those shown in equation (30). Then, with the assumption that neither $\mathscr{V}_{\mathrm{A}}$ nor $\mathscr{V}_{\mathrm{B}}$ contribute to $\mathscr{Q}$, under which projectors $\mathrm{P}$ of the type given by equation (34a) are valid, $\mathrm{P}$ can be chosen to be the projection into the space $\mathscr{V}_{\mathrm{A}+\mathrm{B}}$ spanned by all the vectors of $\mathscr{V}_{\mathrm{A}}$ and $\mathscr{V}_{\mathrm{B}}$ combined; that is, by defining the subspace

$$
\mathscr{V}_{\mathrm{A}+\mathrm{B}}=\left\{\mathscr{V}_{\mathrm{A}}, \mathscr{V}_{\mathrm{B}}\right\}
$$

the projector $P$ is chosen to be:

$$
P=\Pi_{A+B}
$$

An alternative prescription to that of equation (35a) for the subspace $\mathscr{V}_{\mathrm{A}+\mathrm{B}}$ can be obtained from the following constructive argument. Resolve all vectors of $\mathscr{V}_{\mathrm{B}}$ into two components, one contained in $\mathscr{V}_{\mathrm{A}}$ and the other orthogonal to $\mathscr{V}_{\mathrm{A}}$. The set of components in $\mathscr{V}_{\mathrm{A}}$ span a subspace (lying entirely in $\mathscr{V}_{\mathrm{A}}$ ), which is the projection of $\mathscr{V}_{\mathrm{B}}$ into $\mathscr{V}_{\mathrm{A}}$. The set of orthogonal components spans a subspace, called $\mathscr{V}_{\mathrm{BpA}}$, that is orthogonal to $\mathscr{V}_{\mathrm{A}}$. Then $\mathscr{V}_{\mathrm{A}+\mathrm{B}}$ combination of a vector in orthogonal vectors, one in

is the vector sum of $\mathscr{V}_{\mathrm{A}}$ and $\mathscr{V}_{\mathrm{BpA}}$, because any linear $\mathscr{V}_{\mathrm{A}}$ and a vector in $\mathscr{V}_{\mathrm{B}}$ can be expressed as the sum of two $\mathscr{V}_{\mathrm{A}}$ and the other in $\mathscr{V}_{\mathrm{BpA}}$ :

$$
\begin{aligned}
\mathscr{V}_{\mathrm{A}+\mathrm{B}} & =\mathscr{V}_{\mathrm{A}}^{\oplus} \mathscr{V}_{\mathrm{BpA}}=\mathscr{V}_{\mathrm{B}}^{\oplus} \mathscr{V}_{\mathrm{ApB}} \\
\Pi_{\mathrm{A}+\mathrm{B}} & =\Pi_{\mathrm{A}}+\Pi_{\mathrm{BpA}}, \Pi_{\mathrm{A}} \Pi_{\mathrm{BpA}}=0 \\
& =\Pi_{\mathrm{B}}+\Pi_{\mathrm{ApB}}, \Pi_{\mathrm{B}} \Pi_{\mathrm{ApB}}=0
\end{aligned}
$$


If the incident channel is of the $\alpha$ type, the form given by equation (37) corresponds to equation (34a), but, if it is a $\beta$ type, equation (38) corresponds to equation (34a). It depends on the details of a given reaction whether or not $\Pi_{A} \Psi$ arising from the use of equation (37) contributes to the asymptotic behavior in the $\beta$ channels, and likewise for $\Pi_{B} \Psi$ in the $\alpha$ channels when the form of equation (38) is used.

Actually to construct projection operators like $\Pi_{\mathrm{BpA}}$ in a given problem may not be easy, as can be appreciated from the example of stripping or pickup reactions discussed in appendix C. A possible simplification in its use is seen upon writing

$$
\Pi_{\mathrm{BpA}} \Psi=\Pi_{\mathrm{BpA}} \Pi_{\mathrm{B}} \Psi+\Pi_{\mathrm{BpA}}\left(1-\Pi_{\mathrm{B}}\right) \Psi
$$

and noting that only the first term on the right enters into the asymptotic contribution of $\Pi_{\mathrm{BpA}} \Psi$ in the open $\beta$ channels. The projected vector $\left(1-\Pi_{B}\right) \Psi$ contains no such asymptotic information whatever, nor does any further projection of this vector. Equation (37) shows that

$$
\Pi_{\mathrm{A}+\mathrm{B}} \Pi_{\mathrm{B}}=\Pi_{\mathrm{B}} \Pi_{\mathrm{A}+\mathrm{B}}=\Pi_{\mathrm{B}}
$$

and, after inserting equation (37) for $\Pi_{A+B}$ '

$$
\Pi_{B p A} \Pi_{B}=\left(1-\Pi_{A}\right) \Pi_{B}
$$

is obtained. This operator is not itself a projection operator, nor does it yield one when added to $\Pi_{A^{*}}$ (The only exception to this statement occurs when $\Pi_{\mathrm{BpA}}$ and $\Pi_{\mathrm{B}}$ commute, which corresponds to the special case that $\mathscr{V}_{\mathrm{B}}$ is orthogonal to $\mathscr{V}_{\mathrm{A}}$ and, hence, is the same as $\mathscr{V}_{\mathrm{BpA}}{ }^{\text {) }}$ ) The operator (eq. (39)) is easily constructed, however, and when solving equation (24) only that part of $\Pi_{\mathrm{BpA}} \Psi$ given by it needs to be obtained.

When more than two kinds of channels are open, the form of $\mathbf{P}$ given by equations (37) and (38) can be generalized in terms of projections into additional orthogonal subspaces. The alternative expressions for $\mathbf{P}$ reveal that a projector of the form of equation (34a) can also be written as

$$
\begin{aligned}
P & =\Pi_{B}+\Pi_{O^{\prime}}, \Pi_{B^{\prime}} \Pi_{O^{\prime}}=0 \\
& =\Pi_{C}+\Pi_{O^{\prime \prime}}, \Pi_{C^{\prime \prime}}{ }_{O^{\prime \prime}}=0 \text {, etc. }
\end{aligned}
$$

where $\Pi_{\mathbf{B}}$ is the projection into the open $\beta$ channels, $\Pi_{\mathbf{C}}$ is the projection into the 
open $\gamma$ channels, etc. These alternative forms of $\mathbf{P}$ are not required here. They correspond to a multicomponent form of the projector given recently by Hahn (ref. 15) for application to multichannel processes.

\section{Generalized Optical Potentials}

The generalized potentials that describe the scattering into a selected set I of $\alpha$ channels, which includes the incident channel $\alpha$ o as well as an arbitrary group of inelastic channels, may now be derived. For the projection operator $\mathbf{P}$ into all the open channels the one given in equation (34a) is used. The projector $\Pi_{A}$ defined by equation (32) can be resolved as follows:

$$
\begin{gathered}
\Pi_{\mathrm{A}}=\Pi_{\mathrm{I}}+\Pi_{\mathrm{A}-\mathrm{I}} \\
\Pi_{\mathrm{I}^{\Pi} \mathrm{A}-\mathrm{I}}=\Pi_{\mathrm{I}} \Pi_{\mathrm{O}}=\Pi_{\mathrm{A}-\mathrm{I}} \Pi_{\mathrm{O}}=0
\end{gathered}
$$

Both $\Pi_{\mathbf{I}}$ and $\Pi_{\mathrm{A}-\mathrm{I}}$ commute with $\mathrm{K}_{\alpha}$, but as already indicated the projector $\Pi_{\mathrm{O}}$ does not. Combining equations (34a) and (40a) yields

$$
\mathbf{P}=\Pi_{\mathbf{I}}+\Pi_{\mathbf{K}}, \Pi_{\mathbf{I}} \Pi_{\mathbf{K}}=\mathbf{0}
$$

where

$$
\Pi_{\mathbf{K}}=\Pi_{\mathrm{A}-\mathbf{I}}+\Pi_{\mathbf{O}}
$$

is the projector which selects all the open channels except those in the set $\mathbf{I}$. The $\mathbf{K}$ channels comprise the excluded open inelastic channels as well as the open rearrangement channels.

In Feshbach's theory, equation (27) expresses the effective Hamiltonian $H$ for $P \Psi$ as a part $H^{\prime}$, which is almost energy independent, plus a resonance term $H_{R^{*}}$ Further details are reviewed in appendix $B$, where $H^{\prime}$ is given by equation (B2) and a suitable expression for $\mathrm{H}_{\mathrm{R}}$ is developed. In terms of the incident channel resolution of given by equation (1), $H^{\prime}$ has the form

$$
H^{\prime}=P K_{\alpha} P+U_{p}
$$

where 


$$
\mathrm{U}_{\mathrm{p}}=\mathbf{P V _ { \alpha }} \mathbf{P}+\mathrm{U}_{\mathrm{D}}
$$

and $U_{D}$ represents the effect of all distant resonances.

The generalized potential or effective Hamiltonian for $\Psi_{I}$, in a form which separates the slow from the rapid energy dependence may now be obtained. For this purpose, equation (24) is written as

$$
\left(E-H^{\imath}\right) P \Psi=H_{R^{P} \Psi}
$$

Operating on equation (42) with $\Pi_{K}$ and solving for $\Psi_{K} \equiv \Pi_{K} \Psi$ yields

$$
\Psi_{K}=\frac{1}{E-H_{K K}^{i}+i \epsilon}\left(H_{K I}^{:} \Psi_{I}+\Pi_{K} H_{R} P \Psi\right)
$$

where

$$
\mathrm{H}_{\mathrm{KI}}^{\prime}=\Pi_{K} \mathrm{H}^{\prime} \Pi_{I} \text {, etc. }
$$

The first term on the right arises from the coupling between the $\mathrm{I}$ and $\mathrm{K}$ channels, and the second represents the direct coupling from the resonance states into the $\mathrm{K}$ channels. When equation (43) is used with the projection of equation (42) by $\Pi_{I}$, the result can be written as

$$
\left(E-h^{(+)}\right) \Psi_{I}=\Omega^{(-) \dagger} \dot{H}_{R} P \Psi
$$

where

$$
\mathrm{h}^{( \pm)}=\mathrm{H}_{\mathrm{II}}^{\mathrm{s}}+\mathrm{H}_{\mathrm{IK}}^{\prime} \frac{1}{\mathrm{E}-\mathrm{H}_{\mathrm{KK}}^{\prime} \pm \mathrm{i} \epsilon} \mathrm{H}_{\mathrm{KI}}^{\prime}
$$

and

$$
\Omega^{( \pm)}=\Pi_{\mathrm{I}}+\Pi_{\mathrm{K}} \frac{1}{\mathrm{E}-\mathrm{H}_{\mathrm{KK}}^{\prime} \pm \mathrm{i} \epsilon} \mathrm{H}_{\mathrm{KI}}^{\prime}
$$

so that

$$
\mathbf{h}^{(+)}=\Omega^{(-) \dagger} \mathrm{H}^{\top} \Pi_{I}
$$


In order to interpret equation (44), the analogous operations are performed on the equation

$$
\left(E-H^{\prime}\right) \psi_{\mathrm{O}}^{( \pm)}=0
$$

where $\psi_{\mathrm{o}}^{(+)}\left(\psi_{\mathrm{m}}^{(+)}\right)$is the outgoing wave solution with incident wave in channel $\alpha_{0}(\alpha \mathrm{m})$, with the result that

$$
\psi_{\mathrm{OK}}^{( \pm)} \equiv \Pi_{\mathrm{K}} \psi_{\mathrm{O}}^{( \pm)}=\frac{1}{\mathrm{E}-\mathrm{H}_{\mathrm{KK}}^{\prime}+\mathrm{i} \epsilon} \mathrm{H}_{\mathrm{K}}^{\prime} \psi_{\mathrm{OI}}^{( \pm)}
$$

and

$$
\left(E-h^{( \pm)}\right) \psi_{\mathrm{OI}}^{( \pm)}=0
$$

Thus,

$$
\psi_{\mathrm{oI}}^{( \pm)} \equiv \Pi_{\mathrm{I}} \psi_{\mathrm{o}}^{( \pm)}
$$

is the eigenstate of $h^{( \pm)}$with the same asymptotic behavior in the I channels as $\psi_{0}^{( \pm)}$ itself. Also, equation (47) shows that

$$
\psi_{\mathrm{o}}^{( \pm)}=\psi_{\mathrm{oI}}^{( \pm)}+\psi_{\mathrm{OK}}^{( \pm)}=\Omega^{( \pm)} \psi_{\mathrm{oI}}^{( \pm)}
$$

The states $\psi_{\mathrm{m}}^{(+)}\left(\psi_{\mathrm{m}}^{(-)}\right)$are orthonormal, but their projections $\psi_{\mathrm{mI}}^{(+)}(\psi \stackrel{\mathrm{mI}}{(-)})$ are not.

Equation (44) for $\Psi_{I}$ is in the desired form. The resonance contributions to $\Psi_{I}$ are isolated on the right side. The first term on the right gives the direct contribution into the I channels from the resonance states, and the second term corresponds to the indirect contribution that arises as a result of the resonant behavior in the $\mathrm{K}$ channels and their coupling to the I channels. Equations (18) and (44) are completely equivalent to one another, but equation (44) has the advantage of exhibiting the resonance contributions explicitly. The expression for $\Psi_{I}$ obtained by solving equation (44) is in the form of a slowly varying part plus a rapidly varying part:

$$
\Psi_{\mathrm{I}}=\psi\left(\stackrel{\mathrm{OI}}{(+)}+\frac{1}{\mathrm{E}-\mathbf{h}^{(+)}+\mathrm{i} \epsilon} \Omega^{(-) \dagger} \mathrm{H}_{\mathrm{R}} \mathrm{P \Psi}\right.
$$


The transition matrix, which follows from equation (50), is

$$
T(I)=T_{p}(I)+\left\langle\psi(-)\left|\Omega^{(-) \dagger} H_{R}\right| P \Psi\right\rangle
$$

The transition matrix element $\mathrm{T}_{\mathrm{p}}(\mathrm{I})$ given by $\psi_{\text {oI }}^{(+)}$is clearly equal to the matrix element $T_{p}$ given by $\psi_{0}^{(+)}$for any of the I channels, incause $\psi_{0}^{(+)}$has the same asymptotic behavior as $\psi_{\mathrm{oI}}^{(+)}$in these channels. The total transition matrix element (eq. (50)) is the same as that which follows from $\mathrm{P \Psi}$ (eq. (B4)). This correspondence can be verified immediately with the help of equation (49), which gives

$$
\psi_{\mathrm{m}}^{(-)}=\Omega^{(-)} \psi(-)
$$

In order to obtain the generalized optical potential for inelastic scattering, the transition amplitude (eq. (51)) is averaged over a suitable energy interval $\Delta E$, as described in detail in appendix $B$. The steps involved are exactly analogous to those which lead from equation (B4) to equation (B10), and the result is

$$
\overline{\mathrm{T}}(\mathrm{I})=\mathrm{T}_{\mathrm{p}}(\mathrm{I})-\mathrm{i} \pi\left\langle\psi_{\mathrm{mI}}^{(-)}\left|\Omega^{(-) \dagger} \mathscr{H}_{\mathrm{PQ}} \bar{\Lambda} \mathscr{H}_{\mathrm{QP}} \Omega^{(+)}\right| \psi_{\mathrm{OI}}^{(+)}\right\rangle
$$

where

$$
\bar{\Lambda}=\frac{\Lambda}{\Delta \mathrm{E}}
$$

and

$$
\Lambda=\sum_{\Delta \mathrm{E}}\left|\varphi_{\mu}\right\rangle\left\langle\varphi_{\mu}\right|
$$

and where the sum runs over all discrete eigenstates $\varphi_{\mu}$ whose eigenvalues $\mathscr{E}_{\mu}$ lie within the interval $\Delta \mathrm{E}$.

The optical model Hamiltonian

$$
\mathrm{h}_{\mathbf{M}}=\mathrm{h}^{(+)}+\mathrm{u}_{\mathbf{C N}}
$$


can be characterized as follows: At energy value $\mathbf{E}$, the eigenstate $\theta^{(+)}$, which satisfies the same boundary conditions as $\Psi_{I}$, gives the transition matrix (eq. (52)). An integral equation satisifed by eigenstates of $h_{M}$ is

$$
{ }_{\theta}^{(+)}=\psi_{\mathrm{oI}}^{(+)}+\frac{1}{\mathrm{E}-\mathrm{h}^{(+)}+\mathrm{i} \epsilon} \mathrm{u}_{\mathrm{CN}^{\theta^{(+)}}}
$$

while a state with the desired transition matrix is

$$
{ }_{\theta}^{(+)}=\psi_{\mathrm{OI}}^{(+)}-\mathrm{i} \pi \frac{1}{\mathrm{E}-\mathrm{h}^{(+)}+\mathrm{i} \epsilon}\left(\Omega^{(-) \dagger} \mathscr{H}_{\mathrm{PQ}} \bar{\Lambda} \mathscr{H}_{\mathrm{QP}} \Omega^{(+)}\right) \psi_{\mathrm{oI}}^{(+)}
$$

Comparing these last two equations shows that

$$
\mathrm{u}_{\mathrm{CN}}=-\mathrm{i} \pi \Omega^{(-) \dagger} \mathscr{H}_{\mathrm{PQ}} \bar{\Lambda}_{\mathrm{QP}} \Omega^{(+)}{ }_{1-\mathrm{i} \pi \frac{1}{\mathrm{E}-\mathrm{h}^{(+)}+\mathrm{i} \epsilon} \Omega^{(-) \dagger} \mathscr{H}_{\mathrm{PQ}} \overline{\bar{\Lambda} \mathscr{H}} \mathrm{QP}^{\Omega^{(+)}}}^{1}
$$

An alternative approach to the desired optical potential is to obtain it directly from the optical potential $H_{M}$ whose eigenstate $\chi^{(+)}$yields the energy-averaged transition amplitudes for all open channels:

$$
\left(E-H_{M}\right) X^{(+)}=0
$$

The potential $\mathrm{H}_{\mathrm{M}}$ has been derived by Feshbach (ref. 2) and is shown in equation (B11). From equations (B12) and (B15), the generalized optical model Hamiltonian for all channels can be written as

$$
\mathrm{H}_{\mathrm{M}}=\mathrm{PK}_{\alpha} \mathbf{P}+\mathrm{U}
$$

where the generlized optical potential $U$ is

$$
\mathbf{U}=\mathbf{P V} \mathbf{Q}_{\alpha} \mathbf{P}+\mathbf{U}_{\mathrm{D}}+\mathrm{U}_{\mathrm{CN}}
$$

and 


$$
\mathrm{U}_{\mathrm{CN}}=-\frac{\mathrm{i} \pi}{\Delta \mathrm{E}} \mathscr{H}_{\mathrm{PQ}} \Lambda \frac{1}{1-\frac{\mathrm{i} \pi}{\Delta \mathrm{E}} \Lambda \mathrm{W} \Lambda} \Lambda \mathscr{H}_{\mathrm{QP}}
$$

The last equation is a more symmetrical version of equation (B15), and $\mathrm{W}$ is defined by equation (B7b).

It is easy to project out of $x^{(+)}$the part that describes the scattering in the selected $\alpha$ channels and to determine the optical model potential which governs this part. It follows as a consequence of there being no closed channels in the optical model description that

$$
\mathbf{P \chi}^{(+)}=\chi^{(+)}
$$

and that

$$
\mathrm{PH}_{\mathrm{M}}=\mathrm{H}_{\mathrm{M}^{\mathrm{P}}}=\mathrm{H}_{\mathrm{M}}
$$

as is easily verified from the explicit expressions for $x^{(+)}$and $H_{M}$. The form of $P$ given in equation (41a) leads to the resolution

$$
x^{(+)}=x_{I}^{(+)}+x_{K}^{(+)}
$$

Operating on equation (B11) with $\Pi_{K}$ and solving for $x_{\dot{K}}^{(+)}$give

$$
\mathrm{X}_{\mathrm{K}}^{(+)}=\frac{1}{\mathrm{E}-\mathrm{H}_{\mathrm{KK}}^{\mathrm{M}}+\mathrm{i} \epsilon} \mathrm{H}_{\mathrm{KI}}^{\mathrm{M}} \mathrm{X}_{\mathrm{I}}^{(+)}
$$

where $\mathrm{H}_{\mathrm{KI}}^{\mathrm{M}}=\Pi_{\mathrm{K}^{\mathrm{H}}} \mathrm{M}^{\Pi} \mathrm{I}$, etc. Using equation (56) in the equation obtained by operating with $\Pi_{I}$ on equation (B11) then yields

$$
\left(E-H_{\Pi I}^{M}-H_{I K}^{M} \frac{1}{E-H_{K K}^{M}+i \epsilon} H_{K I}^{M}\right) X_{I}^{(+)}=0
$$

which is the desired expression.

Two expressions (given by eqs. (53) and (57)) for a generalized optical potential describing the scattering in a selected set of inelastic channels have been derived. The two 
different forms were obtained by reversing the order of the operations of energy averaging the transition amplitude and projecting into the selected channels. There is no a priori reason for these to be identical potentials, since the condition imposed upon them is only that their corresponding scattering states have the same asymptotic behavior. However, they do, in fact, give the same potential. Their equivalence is most easily established by showing that their scattering states are the same everywhere. The proof of this follows directly by operating with the projector $\Pi_{I}$ on equation (B13b) and making use of the identity

$$
\Pi_{I} \frac{1}{E-H^{r}+i \epsilon} \equiv \frac{1}{E-h^{(+)}+i \epsilon} \Omega^{(-) \dagger}
$$

Comparison with equation (54) then shows that $x_{I}^{(+)}=\theta^{(+)}$.

In the development of this section, the commutativity of $\Pi_{I}$ and $K_{\alpha}$ has not been used as yet. Hence, the equations have the same form when equation (33a) is used for the projector $P$. The use of equation (41a), however, does allow for additional simplifications. For example, the terms in equation (57) can be written as

$$
\begin{gathered}
\mathrm{H}_{\mathrm{II}}^{\mathrm{M}}=\mathrm{K}_{\alpha} \Pi_{\mathrm{I}}+\mathrm{U}_{\mathrm{II}} \\
\mathrm{H}_{\mathrm{IK}}^{\mathrm{M}}=\mathrm{U}_{\mathrm{IK}}, \mathrm{H}_{\mathrm{KI}}^{\mathrm{M}}=\mathrm{U}_{\mathrm{KI}} \\
\mathrm{H}_{\mathrm{KK}}^{\mathrm{M}}=\Pi_{\mathrm{K}} \mathrm{K}_{\alpha} \Pi_{\mathrm{K}}+\mathrm{U}_{\mathrm{KK}}
\end{gathered}
$$

with $\mathrm{U}_{\mathrm{IK}}=\Pi_{\mathrm{I}} \mathrm{U}_{\mathrm{K}}$, etc. Equation (57) then becomes

$$
\left(E-K_{\alpha}-U_{I I}-U_{I K} \frac{1}{E-H_{\mathrm{KK}}^{M}+i \epsilon} U_{K I}\right) x_{I}^{(+)}=0
$$

\section{PROPERTIES OF GENERALIZED POTENTIALS}

Because $P \Psi$ is the projection into all open channels, its effective Hamiltonian $H$ is Hermitian as are the separate terms $\mathrm{H}^{\prime}$ and $\mathrm{H}_{\mathrm{R}^{*}}$. Inspection of equations (25) and (B1a) immediately verifies that these operators are indeed Hermitian. The slowly varying part $H^{\prime}$ contains in the terms $P V_{\alpha} P$ and $U_{D}$ the interaction mechanism that 
causes direct transitions to the open channels. The term $U_{D}$ represents the net effect of all virtual transitions from the open channels to the closed and back. The generalized potential in equation (44), which governs $\Psi_{I}$, is not Hermitian, however, as follows from the imaginary term i $\epsilon$ in the denominators of equations (45) and (46). The imaginary terms represent an absorption of the incident particle arising from processes in which the exit channel belongs to the set of excluded open channels $\mathrm{K}$.

The physical significance of the individual terms appearing in equation (44) may now be interpreted. The part $h^{(+)}$, which is slowly varying with energy, is the contribution to the potential from the direct processes. Equation (45) can be written in the form

$$
\mathbf{h}^{(+)}=\mathrm{H}_{\mathrm{II}}^{\prime}+\mathrm{H}_{\mathrm{IK}}^{\prime} \operatorname{Pr} \frac{1}{\mathrm{E}-\mathrm{H}_{\mathrm{KK}}^{\prime}} \mathrm{H}_{\mathrm{KI}}^{\prime}-\mathrm{i} \pi \mathrm{H}_{\mathrm{IK}}^{\prime} \delta\left(\mathrm{E}-\mathrm{H}_{\mathrm{KK}}^{\prime}\right) \mathrm{H}_{\mathrm{KI}}^{\prime}
$$

where $\operatorname{Pr}$ stands for the principal value. Because $H_{\mathrm{II}}^{\prime}$ and $H_{\mathrm{KK}}^{\prime}$ are Hermitian, the first two terms are Hermitian and the third is imaginary. The first term contains $\Pi_{I} V_{\alpha} \Pi_{I}$ and, in addition, $\Pi_{I} U_{D} \Pi_{I}$, which is the result of virtual transitions from the open I channels to the closed channels and back to the I channels. The second term is the off-energy-shell contribution of the propagator and corresponds to virtual transitions through the closed channels, which are connected to the I channels by way of intermediate states belonging to the open channels $\mathrm{K}$. The third term comes from the energyconserving part of the propagator and represents the effect of the direct transitions to the set $\mathbf{K}$ of excluded open channels.

The resonance terms containing $\mathrm{H}_{\mathrm{R}}$ arise from the reaction mechanism which proceeds via real transitions to the discrete compound states of the closed channel subspace. The term $\Pi_{I} \mathrm{H}_{R} \mathrm{P \Psi}$ gives the decay from the compound states directly into the I channels. The imaginary term from the on-energy-shell part of the propagator in equation (46) involves the decays into the $K$ channels. In both the direct and the resonance terms, the imaginary part will tend to increase when the number of inelastic channels assigned to the set $\mathrm{K}$ is increased, because the transitions associated with the propagator term will become more numerous thereby.

The generalized potential $v_{I}^{(+)}$has many of the properties of the single-channel potential derived by Feshbach (ref. 1). Only slight modifications of his discussion are required here; therefore the discussion is limited mainly to stating the corresponding properties. The smallest threshold energy for opening any of the channels not belonging to the set $\mathrm{I}$ is $\epsilon_{\mathrm{K}}$. The abbreviations $\mathscr{H}_{\Theta \Theta}=\Theta_{\mathrm{I}} \mathscr{H} \Theta_{\mathrm{I}}, \mathrm{V}_{\mathrm{I} \Theta}^{\alpha}=\Pi_{\mathrm{I}} \mathrm{V}_{\alpha} \Theta_{\mathrm{I}}$, etc. are also adopted. The eigenstates of $\mathscr{H}_{\Theta \Theta}$ are displayed as follows

$$
\mathscr{H}_{\Theta \Theta} \eta_{\mathrm{m}}=\epsilon_{\mathrm{m}} \eta_{\mathrm{m}}
$$


for the discrete bound eigenstates and

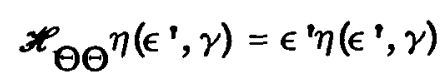

for the continuum eigenstates. The continuous spectrum begins at the minimum value $\epsilon_{K^{*}}$ The additional labels $\gamma$ are those used to designate different states with the same eigenvalue $\epsilon^{\prime}$.

The generalized potentials $H$ and $V_{I}^{(+)}$are nonlocal. This property can be exhibited explicitly by expanding the inverse operator of equation (25) in the complete set of eigenstates of $\mathscr{H}_{\mathrm{QQ}}$ and the inverse operator of equation (17a) in the eigenstates of $\mathscr{H}_{\Theta \Theta}$. In the case of $V_{I}^{(+)}$the result is

$$
\mathrm{v}_{\mathbf{I}}^{(+)}(\mathrm{E})=\mathrm{v}_{\mathrm{II}}^{\alpha}+\sum_{\mathrm{m}} \mathrm{v}_{\mathrm{I} \Theta}^{\alpha} \frac{\left|\eta_{\mathrm{m}}\right\rangle\left\langle\eta_{\mathrm{m}}\right|}{\mathbf{E}-\epsilon_{\mathrm{m}}} \mathrm{v}_{\Theta \mathbf{I}}^{\alpha}+\int \mathrm{d} \gamma \int_{\epsilon_{\mathrm{K}}}^{\infty} \mathrm{d \epsilon ^{ \prime }} \mathrm{v}_{\mathrm{I} \Theta}^{\alpha} \frac{\left|\eta\left(\epsilon^{\prime} \gamma\right)\right\rangle\left\langle\eta\left(\epsilon^{\prime}, \gamma\right)\right|}{\mathbf{E}-\epsilon^{\prime}+\mathbf{i} \epsilon} \mathrm{v}_{\Theta \mathbf{I}}^{\alpha}
$$

In treating the energy dependence of $V_{I}^{(+)}$, the set of channels $I$ is kept fixed, even at small values of $E$ where some of the channels in $I$ are closed. The derivation and formal expression for $V_{I}^{(+)}$are the same at any value of $E$. However, it should be noted that the interpretation of equation (44), as the separation of $v_{I}^{(+)}$into a slowly varying term and a resonant term, becomes invalid when $\Pi_{I}$ includes closed inelastic channels whose projection subspace enters significantly into the description of the compound states with energies close to the value $\mathbf{E}$.

From equation (58),

$$
\begin{aligned}
& \operatorname{ReV}_{\mathbf{I}}^{(+)}(\mathbf{E})=\mathbf{V}_{\mathbf{I I}}^{\alpha}+\sum_{\mathbf{m}} \mathbf{v}_{\mathbf{I} \Theta}^{\alpha} \frac{\left|\eta_{\mathrm{m}}\right\rangle\left\langle\eta_{\mathrm{m}}\right|}{\mathbf{E}-\epsilon_{\mathrm{m}}} \mathbf{v}_{\Theta \mathbf{I}}^{\alpha}+ \\
& +\operatorname{Pr} \int_{\epsilon_{\mathbf{K}}}^{\infty} \frac{\mathrm{d} \epsilon^{\prime}}{\mathrm{E}-\epsilon^{\prime}} \int \mathrm{d} \gamma \mathrm{v}_{\mathrm{I}}^{\alpha}\left|\eta\left(\epsilon^{\prime}, \gamma\right)\right\rangle\left\langle\eta\left(\epsilon^{\prime}, \gamma\right)\right| \mathrm{v}_{\Theta I}^{\alpha}
\end{aligned}
$$

and 


$$
\begin{aligned}
\operatorname{Im} \mathrm{V}_{\mathrm{I}}^{(+)}(\mathrm{E}) & =-\pi \int \mathrm{d} \gamma \mathrm{v}_{\mathrm{I} \Theta}^{\alpha}|\eta(\mathrm{E}, \gamma)\rangle\langle\eta(\mathrm{E}, \gamma)| \mathrm{v}_{\Theta \mathrm{I}}^{\alpha} \quad \text { if } \mathrm{E}>\epsilon_{\mathrm{K}} \\
& =0 \quad \text { if } \mathrm{E}<\epsilon_{\mathbf{K}}
\end{aligned}
$$

From these expressions, it is clear that the imaginary part of $v_{I}^{(+)}$is nonpositive, as it must be if it is to represent an absorption, and that the energy derivative of the real part is nonpositive. A dispersion relation between the real and imaginary parts follows directly from equations (59a) and (59b):

$$
\operatorname{ReV}_{\bar{I}}^{(+)}(\mathrm{E})=\mathrm{v}_{\mathrm{II}}^{\alpha}+\sum_{\mathrm{m}} \mathrm{v}_{\mathrm{I} \Theta}^{\alpha} \frac{\left|\eta_{\mathrm{m}}\right\rangle\left\langle\eta_{\mathrm{m}}\right|}{\mathrm{E}-\epsilon_{\mathrm{m}}} \mathrm{V}_{\Theta \mathrm{I}}^{\alpha}-\frac{1}{\pi} \operatorname{Pr} \int_{\epsilon_{\mathrm{K}}}^{\infty} \frac{\operatorname{Im} V_{\mathrm{I}}^{(+)}\left(\epsilon^{\prime}\right)}{\mathrm{E}-\epsilon^{\prime}} \mathrm{d} \epsilon^{\prime}
$$

The optical model Hamiltonians given by equations (53) and (B12) are, of course, not Hermitian. The spread in energy introduced by the energy averaging allows for an interpretation in terms of a time-dependent wave packet picture. The optical potential describes only the direct or prompt processes accompanying the scattering of the wave packet. The time-delayed part of the wave packet resulting from the formation and subsequent decay of the compound system is not contained in the solution of equation (B11). The averaged effect of all the resonances on the direct reactions is given by $U_{\mathrm{CN}}$, the imaginary part of which accounts for the attenuation of the packet due to the effective absorption of direct particle flux into the compound-state reaction mechanism. In the limit that the matrix elements of $\mathrm{W}$ are small, $\mathrm{U}_{\mathrm{CN}}$ is pure imaginary.

The term ${ }^{{ }_{C N}}$ given by equation (55) similarly serves to give the effect of the resonances in the generalized optical potential (eq. (53)). Its form differs from that of $\mathrm{U}_{\mathrm{CN}}$ because it operates on the projected wave packet, which describes the scattering in the I channels only. However, the compound states are reached by transitions emanating from both the projected part and the projected-out part of the wave packet, and, likewise, the decay of the compound states feeds into both sets of channels. The form of $\mathrm{u}_{\mathrm{CN}}$ takes account of these effects. It is also clear that $H_{M}$ and $h_{M}$ are nonlocal potentials. In the set of coupled equations for the relative motion in the inelastic channels, the matrix elements 


$$
\left\langle\phi_{\alpha \mathrm{m}}\left|\mathrm{h}^{(+)}+\mathrm{u}_{\mathrm{CN}}\right| \phi_{\alpha \mathrm{n}}\right\rangle
$$

enter as coefficients. These are complex functions of the relative coordinates.

\section{Lewis Research Center,}

National Aeronautics and Space Administration, Cleveland, Ohio, February 14, 1967, 129-02-07-07-22. 


\section{APPENDIX A}

\section{SYMBOLS}

A set of all open $\alpha$ channels

d target nucleus consisting of nucleons $1,2, \ldots, \mathrm{A}$

B $\quad$ set of all open $\beta$ channels

$\mathrm{b}_{\mathrm{mn}}\left(\overrightarrow{\mathrm{r}}_{\mathrm{o}}\right) \quad\left(\mathrm{g} \mid \mathrm{Z}_{\mathrm{mn}}\right)_{\vec{\xi}_{1}}$

$c_{n}\left(r_{0}\right) \quad\left(g \mid \lambda_{n}\right)_{\vec{\xi}_{1}}$

$c_{0} \quad\left[(\mathrm{~g} / \mathrm{g})_{\vec{r}_{1}}\right] 1 / 2$

D composite particle consisting of nucleon 1 bound to nucleon $n$

E total energy

$\Delta \mathrm{E} \quad$ energy interval

$\mathbf{E}_{\mathbf{i}} \quad$ eigenvalue of $\mathbf{M}\left(\mathscr{H}_{\mathbf{Q Q}}+\mathrm{W}\right) \mathbf{M}$

$\mathscr{E}_{\nu} \quad$ discrete eigenvalue of $\mathscr{H}_{\mathrm{QQ}}$

$\mathrm{f}_{\mathrm{i}}\left(\overrightarrow{\mathrm{r}}_{\mathrm{d}}\right) \quad\left(\psi_{\mathrm{D}} \mid \mathrm{x}_{\mathrm{i}}\right)_{\vec{\eta}}$

$\mathrm{f}_{\alpha \mathrm{m}} \quad$ coefficient of $\varphi_{\alpha \mathrm{m}}$ in expansion of $\Psi$ in eigenstates of $\mathscr{X}_{\alpha}$

$\mathrm{f}_{\beta \mathrm{m}} \quad$ coefficient of $\varphi_{\beta \mathrm{m}}$ in expansion $\Psi$ in eigenstates of $\mathscr{x}_{\beta}$

G(E) Green's operator

$\overrightarrow{\mathrm{g}}\left(\overrightarrow{\mathrm{r}}_{\mathrm{d}}, \vec{\eta}\right) \quad \mathrm{g}\left(\overrightarrow{\mathrm{r}}_{\mathrm{o}}, \vec{\xi}_{1}\right)$

$\mathrm{g}\left(\overrightarrow{\mathrm{r}}_{\mathrm{o}}, \vec{\xi}_{1}\right) \quad\left(\psi_{\mathrm{o}} \mid \phi_{\mathrm{o}}\right)_{\vec{\xi}_{2}} \ldots, \vec{\xi}_{\mathrm{A}}$

$\mathrm{H}$ effective Hamiltonian for $\mathrm{P} \Psi$

$\mathrm{H}^{\text {? }}$ part of $\mathrm{H}$ which varies slowly with $\mathrm{E}$

$\mathrm{H}_{\mathrm{IK}}^{\mathrm{M}} \quad \Pi_{\mathbf{I}} \mathrm{H}_{\mathbf{M}^{\Pi}} \mathrm{K}_{\mathbf{K}}$

$\mathrm{H}_{\mathrm{IK}}^{\prime} \quad \Pi_{\mathrm{I}^{\mathrm{H}}}{ }^{\prime} \Pi_{\mathrm{K}}$

$\mathrm{H}_{\mathrm{M}} \quad$ optical model Hamiltonian for all open channels

$\mathrm{H}_{\mathrm{R}}$ part of $\mathrm{H}$ which varies rapidly with $\mathbf{E}$ 


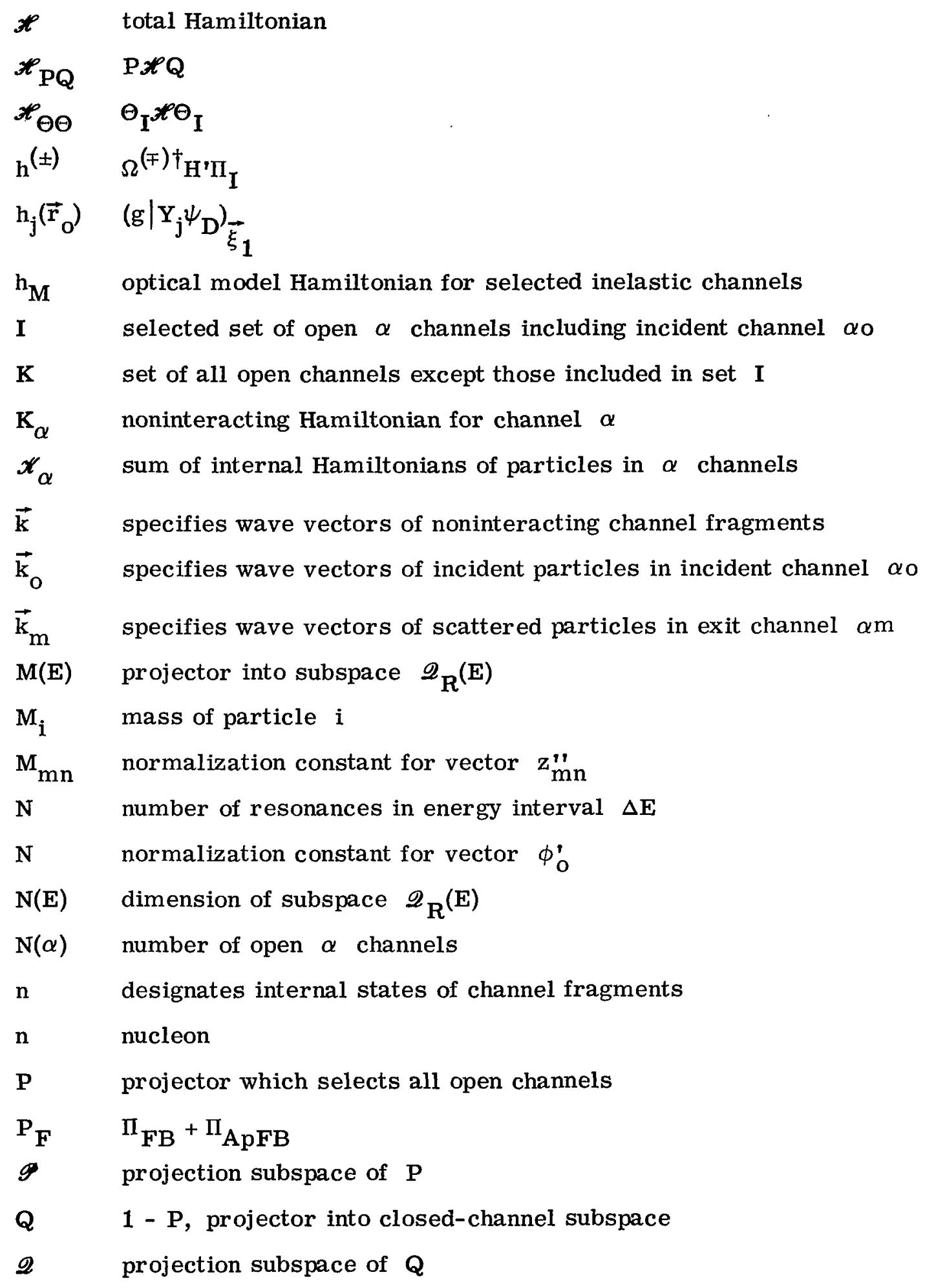


$\mathscr{Q}^{(\mathrm{E})}$

$\mathbf{R}_{\mathrm{A}}$

$\overrightarrow{\mathbf{r}}_{\mathrm{D}}$

$\vec{r}_{i}$

$\mathrm{T}(\mathrm{I})$

$\mathrm{T}\left(\alpha \mathrm{m} \overrightarrow{\mathrm{k}}_{\mathrm{m}} \mid \alpha \circ \overrightarrow{\mathrm{k}}_{\mathrm{o}}\right)$

$\mathrm{T}_{\alpha}$

$\mathrm{T}_{\mathrm{p}}$

$\mathrm{U}$

$\mathrm{U}_{\mathrm{CN}}$

$\mathrm{U}_{\mathrm{D}}$

$\mathrm{U}_{\mathrm{I}}^{(+)}$

$\mathrm{U}_{\text {IK }}$

$\mathrm{U}_{\mathrm{p}}$

$\mathrm{u}_{\mathrm{CN}}$

$\mathrm{u}_{\alpha \overrightarrow{\mathrm{k}}}$

$\mathrm{v}_{\mathrm{I}}^{(+)}$

$\mathrm{v}_{\mathrm{I} \Theta}^{\alpha}$

$\mathrm{v}_{\alpha}$

$v_{A}$

$\mathscr{V}_{\mathrm{A}}$

$\mathscr{V}_{\mathrm{A}}^{(\alpha)}$

$\gamma_{A+B}$ projection subspace of $\mathrm{M}(\mathrm{E})$; space spanned by all $\phi_{\nu}$ whose levels $\mathscr{E}_{\nu}$ overlap at energy $\mathbf{E}$

an effective radius for nucleus $\mathrm{A}$

position vector of particle $\mathrm{D}$ relative to center-of-mass of total system

position vector of particle $i$ relative to center-of-mass of total system transition matrix element for scattering into set of channels I

transition matrix element connecting incident channel $\alpha_{0}$ to exit channel $\alpha \mathrm{m}$

sum of center-of-mass kinetic energies of particles in $\alpha$ channels transition matrix element which follows from $\mathrm{H}^{\prime}$

generalized optical potential

contribution to $\mathrm{H}_{M}$ from averaging over resonances in energy internal $\Delta \mathrm{E}$

contribution of distant resonances to $\mathrm{H}^{\prime}$

operator satisfying $\mathrm{V}_{\alpha} \Psi=\mathrm{U}_{\mathrm{I}}^{(+)} \Psi_{I}$

$\Pi_{\mathbf{I}} \Pi_{\mathbf{K}}$

$\mathrm{PV}_{\alpha} \mathrm{P}+\mathrm{U}_{\mathrm{D}}$

contribution to $h_{m}$ from averaging over resonances in energy internal $\Delta \mathrm{E}$

eigenstate of $T_{\alpha}$ which is product of plane waves with wave vectors specified by $\overrightarrow{\mathrm{k}}$

generalized potential for selected inelastic channels

$\Pi_{I} \mathrm{~V}_{\alpha} \Theta_{\mathbf{I}}$

interaction between particles in $\alpha$ channels

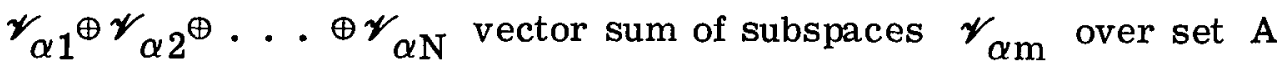

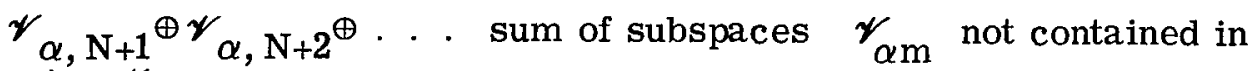
in $\mathscr{\gamma}_{\mathrm{A}}$

$\mathscr{\gamma}_{\alpha 1}^{(\alpha)_{\oplus}} \underset{\alpha 2}{(\alpha)^{\oplus}} \ldots \oplus_{\alpha \mathrm{N}}^{(\alpha)}$

subspace spanned by all vectors of $\mathscr{V}_{\mathrm{A}}$ and $\mathscr{V}_{\mathrm{B}}$ combined 


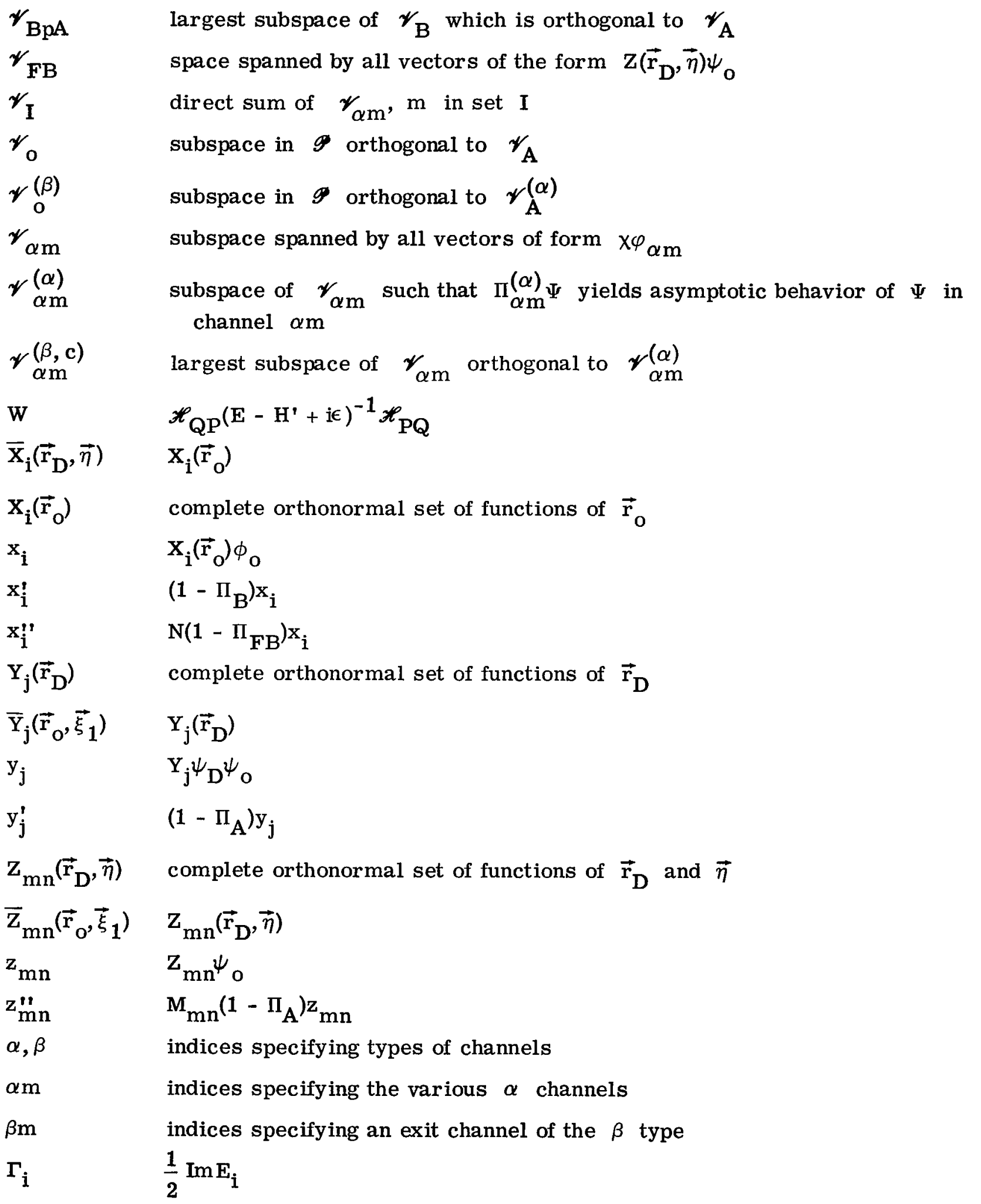


$\epsilon \quad$ positive infinitesimal

$\epsilon_{\mathrm{K}} \quad$ smallest threshold energy for opening up any of the channels not in the set I

$\begin{array}{ll}\vec{\zeta}_{\mathbf{i}} & \text { position } \\ \vec{\eta} & \vec{\eta}_{1}-\vec{\eta}_{\mathrm{o}}\end{array}$

$\eta(\epsilon, \gamma) \quad$ continuum eigenstate of $\mathscr{H}_{\Theta \Theta}$

$\eta_{\mathrm{m}} \quad$ discrete bound eigenstate of $\mathscr{H}_{\Theta \Theta}$

$\vec{\eta}_{1}, \vec{\eta}_{\mathrm{o}} \quad$ position vectors of particle 1 and incident nucleon respectively from center of mass of nucleus $\mathrm{D}$

$\Theta_{\mathbf{I}} \quad 1-\Pi_{\mathbf{I}}$

$\theta^{(+)} \quad$ continuum eigenstate of $h_{m}$

$\Lambda \quad \sum_{\Delta \mathrm{E}}\left|\phi_{\mu}\right\rangle\left\langle\phi_{\mu}\right|$

$\bar{\Lambda} \quad(\Delta \mathrm{E})^{-1} \Lambda$

$\lambda_{n}\left(\vec{\xi}_{1}\right)$ complete orthonormal set in variable $\vec{\xi}_{1}$

$\vec{\xi}_{1} \quad$ position vector of particle $i$ relative to center of mass of nucleus $\mathscr{A}$

$\Pi_{\mathrm{A}} \quad$ projection operator into subspace $\mathscr{V}_{\mathrm{A}}$

$\Pi_{\mathrm{A}}^{(\alpha)}$ projection operator into subspace $\mathscr{V}_{\mathrm{A}}^{(\alpha)}$

$\Pi_{\mathrm{A}-\mathrm{I}} \quad \Pi_{\mathrm{A}}-\Pi_{\mathrm{I}}$

$\Pi_{\mathrm{A}+\mathrm{B}} \quad$ projection operator into subspace $\mathscr{V}_{\mathrm{A}+\mathrm{B}}$

$\Pi_{\mathrm{BpA}}$ projection operator into subspace $\mathscr{V}_{\mathrm{BpA}}$

$\Pi_{\text {FB }}$ projection operator into subspace $\mathscr{V}_{\text {FB }}$

$\Pi_{\mathrm{I}} \quad \sum_{\mathrm{m} \subset \mathrm{I}} \Pi_{\alpha \mathrm{m}}$

$\Pi_{\mathrm{K}} \quad \Pi_{\mathrm{A}-\mathrm{I}}+\Pi_{\mathrm{o}}$

$\Pi_{0} \quad$ projection operator into subspace $\mathscr{V}_{\mathrm{o}}$

$\Pi_{0}^{(\beta)} \quad$ projection operator into subspace $\mathscr{V}_{0}^{(\beta)}$

$\Pi_{\alpha \mathrm{m}} \quad$ projection operator into subspace $\mathscr{V}_{\alpha \mathrm{m}}$

$\Pi_{\alpha \mathrm{m}}^{(\alpha)} \quad$ projection operator into subspace $\mathscr{V}_{\alpha \mathrm{m}}^{(\alpha)}$ 
$\Pi_{\alpha \mathrm{m}}^{(\beta, C)}$ projection operator into subspace $\mathscr{V}_{\alpha \mathrm{m}}^{(\beta C)}$

$\varphi_{\alpha \mathrm{n}} \quad$ eigenstate of $\mathscr{x}_{\alpha}$

$\varphi_{\alpha \mathbf{n} \overrightarrow{\mathbf{k}}} \quad$ eigenstate of $\mathbf{K}_{\alpha}$

$\phi_{0} \quad$ ground state of nucleus $\mathscr{A}$

$\phi_{0}^{\prime} \quad \mathrm{N}\left[\phi_{0}-\mathrm{g}\left(\overrightarrow{\mathrm{r}}_{1}\right) \psi_{\mathrm{o}}\right]$

$\phi_{\nu} \quad$ discrete eigenstate of $\mathscr{H}_{\mathrm{QQ}}$

$X \quad$ any state of motion for mass centers of particles $\alpha$

$x^{(+)} \quad$ continuum eigenstate of $H_{M}$

$x_{I}^{(+)} \quad \Pi_{I} X^{(+)}$

$\chi_{\mathrm{K}}^{(+)} \quad \Pi_{K^{\prime}} \chi^{(+)}$

$\Psi \quad \Psi_{\alpha \mathrm{ok}}^{(+)}$

$\Psi^{*} \quad \Theta_{I^{\Psi}}$

$\Psi_{I} \quad \Pi_{\mathbf{I}} \Psi$

$\Psi_{\alpha \mathrm{ok}}^{(+)} \quad$ continuum eigenstate of $\mathscr{H}$

$\psi_{\mathrm{D}} \overrightarrow{(\eta)} \quad$ ground state of nucleus $\mathrm{D}$

$\psi_{i} \quad$ discrete eigenfunctions of potential function of mass-of-center coordinates of $\alpha$ particles

$\psi_{\mathbf{i}}(\vec{\eta}) \quad$ complete set of states of nucleus D

$\psi_{\mathrm{m}}^{( \pm)} \quad$ continuum eigenstate of $\mathrm{H}^{\prime}$

$\psi_{\mathrm{o}} \quad$ ground state of nucleus $\mathscr{A}-1$

$\psi_{0}^{( \pm)} \quad$ continuum eigenstate of $H^{\prime}$

$\psi_{\mathrm{OI}}^{( \pm)} \quad \Pi_{\mathrm{I}} \psi_{\mathrm{o}}^{( \pm)}$

$\psi_{\mathrm{oK}}^{( \pm)} \quad \Pi_{\mathrm{K}}{ }_{\mathrm{O}}^{( \pm)}$

$\Omega^{( \pm)} \quad \Pi_{I}+\Pi_{K} \frac{1}{E-H_{K K}^{\prime} \pm \mathrm{i} \epsilon} H_{K I}^{\prime}$

$\omega \quad \mathrm{M}\left(\mathrm{E}-\mathscr{H}_{\mathrm{QQ}}\right)^{-1} \dot{\mathscr{H}}_{\mathrm{QP}}|\mathrm{P} \Psi\rangle$ 


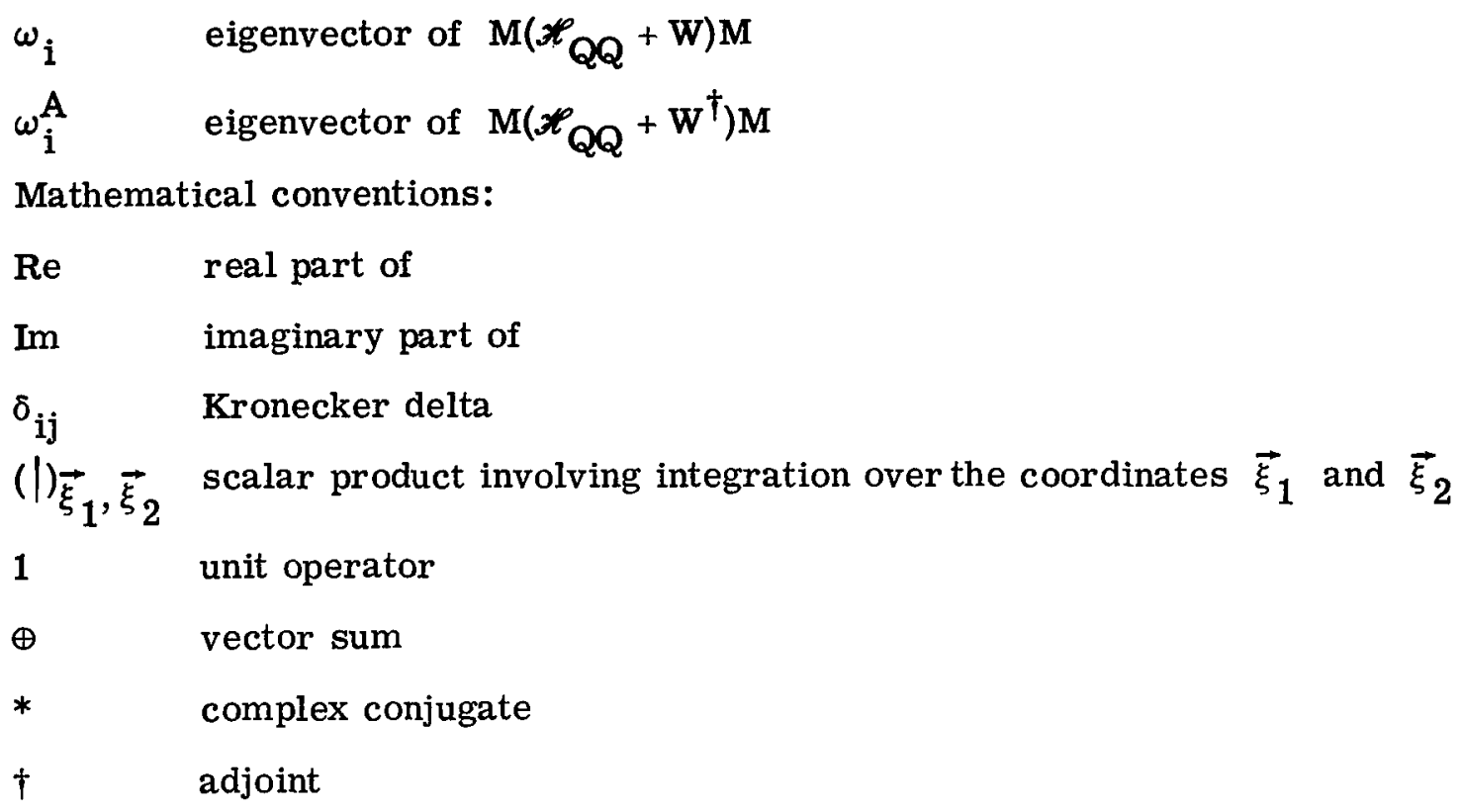




\section{APPENDIX B}

\section{REVIEW OF PERTINENT CONCEPTS FROM FESHBACH'S \\ UNIFIED THEORY OF NUCLEAR REACTIONS}

When the inverse operator in equation (25) is expanded in the complete set of eigenstates of $\mathscr{H}_{\mathrm{QQ}}$, the energy dependence of $\mathbf{H}$ in the neighborhood of $\mathbf{E}$ can be expressed as the sum of a slowly varying part and a rapidly varying part:

$$
\mathscr{H}_{\mathrm{PQ}} \frac{1}{\mathrm{E}-\mathscr{H}_{\mathrm{QQ}}} \mathscr{H}_{\mathrm{QP}}=\mathscr{H}_{\mathrm{PQ}} \sum_{\mu} \frac{\left|\phi_{\mu}\right\rangle\left\langle\phi_{\mu}\right|}{\mathrm{E}-\mathscr{E}_{\mu}} \mathscr{H}_{\mathrm{QP}}+\mathrm{U}_{\mathrm{D}}
$$

In the first term on the right, which is called $H_{R}$, the sum extends over the discrete levels $\mathscr{E}_{\mu}$, which are sufficiently close to the value $\mathbf{E}$ that their contributions vary rapidly. The term $U_{D}$ represents the remaining terms from the sum over the complete set. It is the net contribution of all virtual transitions from the open channels to the distant resonances and back and varies slowly with $E$. The strongly energy-dependent part of $\mathrm{H}$ can be written as

$$
\mathrm{H}_{\mathrm{R}}=\mathscr{H}_{\mathrm{PQ}} \mathrm{M}(\mathrm{E}) \frac{1}{\mathrm{E}-\mathscr{H}_{\mathrm{QQ}}} \mathscr{H}_{\mathrm{QP}}
$$

where

$$
\mathrm{M}(\mathrm{E})=\sum_{\mu}\left|\phi_{\mu}\right\rangle\left\langle\phi_{\mu}\right|
$$

The set of $\mu$ values in the summation, called $\mathrm{N}(\mathrm{E})$ in number, depends on the value of $E$. In the region of isolated resonances, $N(E)$ is at most equal to 1 . Note that $M(E)$ is the projector into the $\mathrm{N}(\mathrm{E})$ dimensional subspace $\mathscr{Q}_{\mathbf{R}}(\mathrm{E})$ of $\mathscr{Q}$ spanned by the corresponding set of orthonormal vectors $\phi_{\mu}$. The remaining part of $\mathrm{H}$ is the slowly varying part $\mathrm{H}^{\prime}$, so that

$$
\mathrm{H}^{\prime}=\mathscr{H}_{\mathrm{PP}}+\mathrm{U}_{\mathrm{D}}
$$


The integral equation for $\mathrm{P} \Psi$ in terms of these two parts of $\mathrm{H}$ is

$$
\mathrm{P} \Psi=\psi_{\mathrm{o}}^{(+)}+\frac{1}{\mathrm{E}-\mathrm{H}^{\prime}+\mathrm{i} \epsilon} \mathrm{H}_{\mathrm{R}} \mathrm{P \Psi}
$$

where $\psi_{0}^{(+)}$is the eigenstate of $\mathrm{H}^{\prime}$ with the same boundary conditions as $\Psi$. The amplitude for the transition from the incident channel $\alpha$ o to the open channel $\alpha \mathrm{m}$ is

$$
\mathrm{T}\left(\alpha \mathrm{m} \overrightarrow{\mathrm{k}}_{\mathrm{m}} \mid \alpha \mathrm{ok} \overrightarrow{\mathrm{o}}_{\mathrm{o}}\right)=\mathrm{T}_{\mathrm{p}}+\left\langle\psi_{\mathrm{m}}^{(-)} \mathrm{H}_{\mathrm{R}} \mid \mathrm{P \Psi}\right\rangle
$$

where $\vec{k}_{o}$ and $\vec{k}_{m}$ designate, respectively, the incident and final wave vectors of the $\alpha$ particles and $\psi_{\mathrm{m}}^{(-)}$is the eigenstate of $\mathrm{H}^{\prime}$ with the energy value $\mathrm{E}$, and the boundary conditions of incoming spherical waves in the open channels and plane waves in channel $\alpha \mathrm{m}$ with wave vectors specified by $\overrightarrow{\mathrm{k}}_{\mathrm{m}}$. Here, $\mathrm{T}_{\mathrm{p}}$ is the transition matrix element which follows from $\psi_{0}^{(+)}$.

There is a rapid energy dependence from the second term on the right of equation (B4), and it originates from both $\mathrm{H}_{\mathrm{R}}$ and $\mathrm{P \Psi}$. (It is assumed that the energy is not in the neighborhood of a threshold for opening a new channel, so the projection operator $\mathbf{P}$ does not change with energy.) In order to express the resonance variation explicitly, the rapidly varying part is written as

$$
\mathrm{H}_{\mathrm{R}} \mathrm{P \Psi}=\mathscr{*}_{\mathrm{PQ}}^{\omega}
$$

and a more suitable form of the vector

$$
\omega=\mathrm{M}(\mathrm{E})\left(\mathrm{E}-\mathscr{H}_{\mathrm{QQ}}\right)^{-1} \mathscr{H}_{\mathrm{QP}}|\mathrm{P} \Psi\rangle
$$

is obtained. From equation (23), $\omega$ is seen to be the projection of $Q \Psi$ into $\mathscr{Q}_{\mathrm{R}}(\mathrm{E})$. When equation (B3) is used in the defining equation (B5) and the commutativity of $\mathscr{H}_{\mathrm{QQ}}$ and $M(E)$ is used, an equation for $\omega$ results that can be expressed as

$$
\left(\mathrm{E}-\mathscr{H}_{\mathrm{QQ}}-\mathrm{MWM}\right) \omega=\mathrm{M} \mathscr{H}_{\mathrm{QP}} \psi_{\mathrm{O}}^{(+)}
$$

where

$$
\mathrm{W}=\mathscr{H}_{\mathrm{QP}} \frac{1}{\mathrm{E}-\mathrm{H}^{2}+\mathrm{i} \epsilon} \mathscr{H}_{\mathrm{PQ}}
$$


Equation (B6a) is a linear equation in the subspace $\mathscr{Q}_{R}(E)$ and is conveniently solved in terms of its Green's operator:

$$
G(E)=\sum_{i=1}^{N(E)} \frac{\left|\omega_{i}\right\rangle\left\langle\omega_{i}^{A}\right|}{E-E_{i}}
$$

Here, $\omega_{i}$ is the eigenvector of $\mathbf{M}\left(\mathscr{H}_{\mathbf{Q Q}}+\mathbf{W}\right) \mathbf{M}$ with the eigenvalue $\mathbf{E}_{\mathbf{i}}$, and $\omega_{\mathbf{i}}^{\mathbf{A}}$ is the corresponding eigenvector of $\mathbf{M}\left(\mathscr{t}_{\mathrm{QQ}}+\mathrm{W}^{\dagger}\right) \mathbf{M}$ with eigenvalue $\mathrm{E}_{\mathbf{i}}^{*}$. The two types of vectors form a complete biorthogonal set in the subspace $\mathscr{Q}_{\mathbf{R}}{ }^{(\mathbf{E}) \text { : }}$

$$
\begin{gathered}
\left\langle\omega_{i}^{\mathrm{A}} \mid \omega_{j}\right\rangle=\delta_{i j} \quad E_{i} \neq E_{j} \\
\sum_{i}\left|\omega_{i}\right\rangle\left\langle\omega_{i}^{\mathrm{A}}\left|=\sum_{\mu}\right| \varphi_{\mu}\right\rangle\left\langle\varphi_{\mu}\right|
\end{gathered}
$$

Then the desired expression for $\omega$ is

$$
\omega=\mathrm{G}(\mathrm{E}) \mathscr{H}_{\mathrm{QP}}{ }_{\mathrm{O}}^{(+)}
$$

for, when this is inserted into equation (B4), the transition amplitude becomes

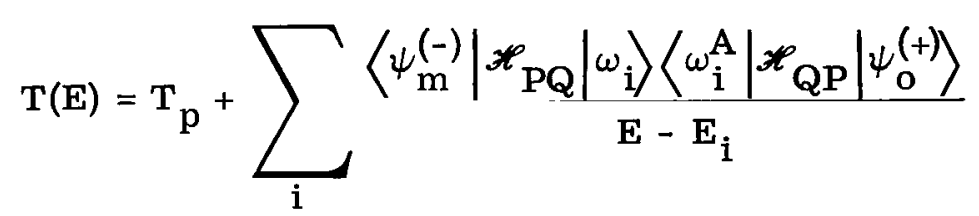

In this form, the energy variation of $T$ in the neighborhood of $E$ arises almost entirely from the demoninators in the second term. Let $\Gamma_{i}$ be one-half the imaginary part of $E_{i}$. It is assumed that $\Gamma_{i} \ll \operatorname{Re}\left(E_{i}\right)$. It is also assumed that $E_{i}$ and $\omega_{i}$ have only a slight energy dependence in an interval of the order of $\Gamma_{i}$ about the energy value $\operatorname{Re}\left(E_{i}\right)$. Then it is meaningful to speak of an individual resonance $E_{i}$, even in the overlapping resonances region, and the energy width $\Gamma_{i}$, about the value $\operatorname{Re}\left(E_{i}\right)$, in which it contributes a rapid variation to the transition amplitude.

The transition amplitude is now averaged over an energy interval $\Delta \mathrm{E}$ which is big enough to encompass a large number $N$ of resonances and to be much greater than $\Gamma_{i}$, 
but yet is sufficiently small that $T_{p}$ (or equivalently $H^{\prime}$ ) is essentially constant over the interval. (The term $U_{D}$ in $H^{\prime}$ varies slowly with energy over an energy interval considerably larger than the average value $\left\langle\Gamma_{\mathrm{i}}\right\rangle$, because only the "nearest" distant resonances change with $\mathrm{E}$ and these constitute a small potion of all the distant resonances contributing at any given energy.) It follows from the assumptions made that to a good approximation

$$
\overline{\mathrm{T}}=\mathrm{T}_{\mathrm{p}}-\frac{\mathrm{i} \pi}{\Delta \mathrm{E}} \sum_{\mathrm{i}=1}^{\mathrm{N}}\left\langle\psi_{\mathrm{m}}^{(-)}\left|\mathscr{H}_{\mathrm{PQ}}\right| \omega_{\mathrm{i}}\right\rangle\left\langle\omega_{\mathrm{i}}^{\mathrm{A}}\left|\mathscr{H}_{\mathrm{QP}}\right| \psi_{\mathrm{o}}^{(+)}\right\rangle
$$

The sum over resonances now includes all $\mathbf{E}_{\mathbf{i}}$ lying in $\Delta \mathrm{E}$. As $\mathrm{E}$ varies over the range of integration, a cumulative vector sum of the projective spaces $\mathscr{Q}_{R}{ }^{(E) \text { gives a }}$ total projective space spanned by all the eigenstates $\varphi_{\mu}$ with values of $\mathscr{E}_{\mu}$ laying in $\Delta \mathrm{E}$. The projector for this subspace associated with $\Delta \mathrm{E}$ is

$$
\Lambda=\sum_{\Delta \mathbf{E}}\left|\varphi_{\mu}\right\rangle\left\langle\varphi_{\mu}\right|
$$

Under the assumption that the $\omega_{i}$ are energy independent, both sums in equation (B8b) may be extended to run over $\Delta \mathrm{E}$ which permits equation (B9) to be replaced by

$$
\overline{\mathrm{T}}=\mathrm{T}_{\mathrm{p}}-\frac{\mathrm{i} \pi}{\Delta \mathrm{E}}\left\langle\psi_{\mathrm{m}}^{(-)}\left|\mathscr{H}_{\mathrm{PQ}}{ }^{\Lambda \mathscr{H}} \mathrm{QP}\right| \psi_{\mathrm{o}}^{(+)}\right\rangle
$$

It is clear that the form of expressions like (B10) is the same for either the case of isolated or of overlapping resonances.

The generalized optical model Hamiltonian $\mathrm{H}_{M}$ for all the open channels is defined by the condition that at the energy $\mathrm{E}$ equal to the average in $\Delta \mathrm{E}$, its scattering state $\chi^{(+)}$, which satisfies

$$
\left(\mathrm{E}-\mathrm{H}_{\mathrm{M}}\right) \mathrm{X}^{(+)}=0
$$

with the same boundary conditions as $\mathrm{P} \Psi$, yields the transition matrix $\overline{\mathrm{T}}$. The part $T_{p}$ of $\bar{T}$ corresponds to the scattering given by a state of $H^{\prime}$, so that an additional part $\mathrm{U}_{\mathrm{CN}}$ is appended to $\mathrm{H}^{\prime}$, in order to produce the contribution arising from the averaged 
effect of the resonances, that is, the second term on the right of equation (B10)

$$
\mathbf{H}_{\mathbf{M}}=\mathbf{H}^{\prime}+\mathrm{U}_{\mathrm{CN}}
$$

An integral equation satisfied by $\chi^{(+)}$is obtained from the two-potential form (eq. (B12)) of $\mathrm{H}_{\mathbf{M}}$, namely,

$$
\chi^{(+)}=\psi_{0}^{(+)}+\frac{1}{E-H^{\prime}+\mathbf{i} \epsilon} U_{C N} \chi^{(+)}
$$

On the other hand, an explicit solution for $\chi^{(+)}$, which gives the transition matrix $\vec{T}$, is

$$
\mathrm{X}^{(+)}=\psi_{\mathrm{o}}^{(+)}+\frac{1}{\mathrm{E}-\mathrm{H}^{+}+\mathrm{i} \epsilon}\left(-\frac{\mathrm{i} \pi}{\Delta \mathrm{E}} \mathscr{H}_{\mathrm{PQ}}{ }^{\Lambda} \mathscr{H}_{\mathrm{QP}}\right) \psi_{\mathrm{O}}^{(+)}
$$

Comparing equation (B13a) and (B13b) shows that

$$
\mathrm{U}_{\mathrm{CN}} \mathrm{X}^{(+)}=-\mathrm{i} \pi \mathscr{H}_{\mathrm{PQ}} \bar{\Lambda} \mathscr{H}_{\mathrm{QP}}{ }_{\mathrm{o}}^{(+)}
$$

where $\Lambda=(\Delta E)^{-1} \Lambda$. Inserting the reciprocal form of equation (B13b) into equation (B14) gives

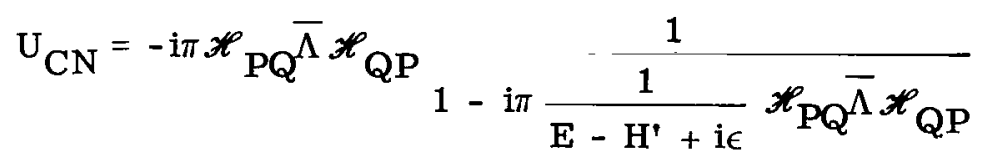




\section{APPENDIX C}

\section{PICKUP PROCESSES}

The projection operators previously developed are applied to a concrete example, namely, for the case where two kinds of open channels are related through a pickup (or stripping) process. Only a single channel of each type is assumed to be open; that is, the internal eigenstate in both channels is that in which all composite fragments occupy their ground states only. The extension to multiple open channels is straightforward. The $\alpha$ fragments consist of the nucleon $\mathrm{n}$ and nucleus $\mathscr{A}$. In the $\beta$ set, particle 1 of nucleus $\mathscr{A}$ is bound to $\mathrm{n}$ forming the composite particle $\mathrm{D}$, while the remaining particles 2,..., A form the nucleus $\mathscr{A}-1$. The following position vectors for particle $\mathrm{i}$ will be employed, where the nucleon $\mathrm{n}$ is labeled by $\mathbf{i}=0$ :

$\vec{r}_{i} \quad$ position vector of particle $i$ measured from center of mass of total system as origin

$\vec{\xi}_{\mathrm{i}} \quad$ from center of mass of nucleus $\mathscr{A}$ as origin

$\vec{\zeta}_{\mathbf{i}} \quad$ from center of mass of nucleus $\mathscr{A}-1$ as origin

$\vec{\eta}_{1}, \vec{\eta}_{\mathrm{o}}$ from center of nucleus $\mathrm{D}$ as origin

$$
\vec{\eta}=\vec{\eta}_{1}-\vec{\eta}_{\mathrm{o}}
$$

The spin variables will not be explicitly indicated. The following kinematical relations between the position vectors are needed. Here, $M_{i}$ is the mass of particle $i$ :

$$
\begin{gathered}
\overrightarrow{\mathrm{r}}_{\mathrm{D}}=\left(\mathrm{M}_{0}+\mathrm{M}_{1}\right)^{-1}\left[\mathrm{M}_{1} \vec{\xi}_{1}+\mathrm{M}_{0}\left(1-\mathrm{M}_{\mathrm{A}}^{-1}\right) \overrightarrow{\mathrm{r}}_{0}\right] \\
\vec{\eta}=\vec{\xi}_{1}-\left(1+\mathrm{M}_{0} \mathrm{M}_{\mathrm{A}}^{-1}\right) \overrightarrow{\mathrm{r}}_{0} \\
\vec{\zeta}_{\mathrm{i}}=\vec{\xi}_{\mathrm{i}}+\frac{\mathrm{M}_{\mathrm{D}}}{\mathrm{M}_{\mathrm{A}-1}} \overrightarrow{\mathrm{r}}_{\mathrm{D}}-\frac{\mathrm{M}_{0}}{\mathrm{M}_{\mathrm{A}}} \overrightarrow{\mathrm{r}}_{0}
\end{gathered}
$$

The natural set of coordinates in the $\alpha$ channel is $\left(\vec{r}_{0}, \vec{\xi}_{1}, \vec{\xi}_{2}, \ldots ., \vec{\xi}_{A}\right)$ and in the $\beta$ channel is $\left(\overrightarrow{\mathrm{r}}_{\mathrm{D}}, \vec{\eta}, \vec{\zeta}_{2}, \ldots, \vec{\zeta}_{\mathrm{A}}\right)$. Equations (C1a) to (C1c) give the linear transformation between them. The internal state wave functions are 


$$
\begin{gathered}
\varphi_{\alpha \mathrm{o}}=\phi_{\mathrm{o}}\left(\vec{\xi}_{1}, \ldots, \vec{\xi}_{\mathrm{A}}\right) \\
\varphi_{\beta \mathrm{o}}=\psi_{\mathrm{D}}(\vec{\eta}) \psi_{\mathrm{o}}\left(\vec{\xi}_{2}, \ldots, \vec{\zeta}_{\mathrm{A}}\right)
\end{gathered}
$$

(The coordinates $\vec{\xi}_{i}$ can be treated as independent if a delta function $\delta\left(\sum \vec{\xi}_{i}\right)$ is included in any integration which involves them, and similarly for the $\vec{\zeta}_{j}$ ) The subspace $\mathscr{V}_{A}$ comprises all functions of the form $\mathbf{x}\left(\vec{r}_{0}\right) \phi_{0}$. An orthonormal basis for the subspace is the set of all

$$
\mathrm{x}_{\mathbf{i}}=\mathrm{x}_{\mathrm{i}} \varphi_{\mathrm{o}}
$$

where the $x_{i}\left(\vec{r}_{0}\right)$ are a complete orthonormal set of functions of $\vec{r}_{0}$ :

$$
\sum_{i} x_{i}\left(\vec{r}_{0}\right) x_{i}^{*}\left(\vec{r}_{0}\right)=\delta\left(\vec{r}_{0}-\vec{r}_{0}^{\prime}\right)
$$

The projector $\Pi_{\mathrm{A}}$ into the space $\mathscr{V}_{\mathrm{A}}$ is given by

$$
\Pi_{A}=\sum\left|x_{i}\right\rangle\left\langle x_{i}\left|=1_{r_{0}}\right| \phi_{0}\right\rangle\left\langle\phi_{0}\right|
$$

Note that $\Pi_{A}$ is proportional to a unit operator in $\vec{r}_{0}$-space. A complete coordinate representation is

$$
\Pi_{\mathrm{A}}=\delta\left(\overrightarrow{\mathbf{r}}_{0}-\overrightarrow{\mathbf{r}}_{0}^{\prime}\right) \phi_{\mathrm{o}}\left(\vec{\xi}_{1}^{\prime}, \ldots, \vec{\xi}_{\mathbf{A}}^{\prime}\right) \phi_{0}^{*}\left(\vec{\xi}_{1}^{\prime}, \ldots, \vec{\xi}_{A}^{\prime}\right)
$$

Similarly, an orthonormal basis for $\mathscr{V}_{B}$ is the set of all

$$
\mathrm{y}_{\mathrm{j}}=\mathrm{Y}_{\mathrm{j}} \psi_{\mathrm{D}} \psi_{\mathrm{o}}
$$

where the completeness of the orthonormal set $Y_{j}$ is expressed by the closure relation

$$
\sum_{j} Y_{j}\left(\vec{r}_{D}\right) Y_{j}^{*}\left(\vec{r}_{D}^{\prime}\right)=\delta\left(r_{D}-r_{D}^{\prime}\right)
$$

The projector $\Pi_{\mathrm{B}}=1_{\vec{r}_{\mathrm{D}}}\left|\psi_{\mathrm{D}} \psi_{\mathrm{o}}\right\rangle\left\langle\psi_{\mathrm{D}} \psi_{\mathrm{o}}\right|$ into $\mathscr{V}_{\mathrm{B}}$ has the representation 


$$
\Pi_{\mathrm{B}}=\delta\left(\overrightarrow{\mathrm{r}}_{\mathrm{D}}-\overrightarrow{\mathrm{r}}_{\mathrm{D}}^{\prime}\right) \psi_{\mathrm{D}}(\vec{\eta}) \psi_{\mathrm{o}}\left(\vec{\zeta}_{2}, \ldots, \vec{\zeta}_{\mathrm{A}}\right) \psi_{\mathrm{D}}^{*}\left(\vec{\eta}^{\prime}\right) \psi_{\mathrm{o}}^{*}\left(\vec{\zeta}_{2}^{\prime}, \ldots, \vec{\zeta}_{\mathrm{A}}\right)
$$

It is convenient to define the function

$$
\left(\psi_{\mathrm{o}}\left(\vec{\xi}_{2}, \ldots, \vec{\zeta}_{\mathrm{A}}\right) \mid \phi_{\mathrm{o}}\left(\vec{\xi}_{1}, \ldots, \vec{\xi}_{\mathrm{A}}\right)\right)_{\xi(2, \ldots, \mathrm{A})}=\overline{\mathrm{g}}\left(\overrightarrow{\mathrm{r}}_{0}, \vec{\xi}_{1}\right) \equiv \mathrm{g}\left(\overrightarrow{\mathrm{r}}_{\mathrm{D}}, \vec{\eta}\right)
$$

where the subscripts indicate the partial set of coordinates that are integrated over in forming the scalar product. Since $\phi_{0}$ is a bound state of nucleus $\mathscr{A}$,

$$
\mathrm{g}\left(\overrightarrow{\mathrm{r}}_{0}, \vec{\xi}_{1}\right) \approx 0 \quad \text { when } \quad\left|\vec{\xi}_{1}\right|>>\mathrm{R}_{\mathrm{A}}
$$

with $R_{A}$ representing some effective radius for the nucleus $\mathscr{A}$. This implies, in turn, that

$$
\mathrm{g}\left(\overrightarrow{\mathrm{r}}_{\mathrm{D}}, \vec{\eta}\right) \rightarrow 0 \quad \text { when } \quad|\vec{\eta}| \lesssim \mathbf{R}_{\mathrm{D}}, \quad\left|\overrightarrow{\mathrm{r}}_{\mathrm{D}}\right| \rightarrow \infty
$$

If the projection $\Pi_{B} x_{i}$ into $\mathscr{V}_{B}$ is substracted from each basis vector $x_{i}$, the resulting set of vectors span the subspace $\mathscr{Y}_{\mathrm{ApB}}$. From equations (C3), (C6), and (C7) there follows the result

$$
\Pi_{\mathrm{B}} \mathrm{x}_{\mathrm{i}}=\mathrm{f}_{\mathrm{i}}\left(\overrightarrow{\mathrm{r}}_{\mathrm{D}}\right) \psi_{\mathrm{D}}(\vec{\eta}) \psi_{\mathrm{o}}\left(\vec{\zeta}_{2}, \ldots, \vec{\zeta}_{\mathrm{A}}\right)
$$

where

$$
\begin{aligned}
\mathrm{f}_{\mathbf{i}}\left(\overrightarrow{\mathrm{r}}_{\mathrm{D}}\right)= & \left(\psi_{\mathbf{D}}(\vec{\eta}) \mid \overline{\mathbf{x}}_{\mathbf{i}}\left(\overrightarrow{\mathrm{r}}_{\mathrm{D}}, \vec{\eta}\right) \overline{\mathrm{g}}\left(\overrightarrow{\mathrm{r}}_{\mathrm{D}}, \vec{\eta}\right)\right)_{\eta} \\
& \overline{\mathbf{x}}_{\mathbf{i}}\left(\overrightarrow{\mathrm{r}}_{\mathrm{D}}, \eta\right) \equiv \mathbf{x}_{\mathbf{i}}\left(\overrightarrow{\mathrm{r}}_{0}\right)
\end{aligned}
$$

Since $\psi_{\mathrm{D}}(\vec{\eta})$ is a bound state, equation (C8b) shows that

$$
f_{i}\left(\vec{r}_{D}\right) \rightarrow 0 \quad \text { when } \quad\left|\vec{r}_{D}\right| \rightarrow \infty
$$

At asymptotic separations in channel $\alpha,\left|\overrightarrow{\mathbf{r}}_{0}\right| \rightarrow \infty,\left|\vec{\xi}_{1}\right| \lesssim \mathbf{R}_{A}$, and, in view of equation (C1a), this in turn implies that $\left|\overrightarrow{\mathrm{r}}_{\mathrm{D}}\right| \rightarrow \infty$ also. Thus, the vectors

$$
x_{i}^{\prime}=\left(1-\Pi_{B}\right) x_{i}
$$


which are orthogonal to $\mathscr{V}_{\mathrm{B}}$ and span $\mathscr{V}_{\mathrm{ApB}}$, have the same asymptotic behavior in channel $\alpha$ as the original basis vectors $\mathrm{x}_{\mathrm{i}}$. An easy calculation yields

$$
\left\langle x_{j}^{!} \mid x_{i}^{i}\right\rangle=\delta_{i j}-\left(f_{j} \mid f_{i}\right)
$$

Hence, the $x_{i}^{\prime}$ are not, in general, orthogonal to one another, and, in fact, need not be a linearly independent set. Of course, the subspace $\mathscr{V}_{\mathrm{ApB}}$ does not depend on the choice of basis functions $\mathrm{X}_{\mathrm{i}}\left(\mathrm{r}_{0}\right)$, and the construction of the subspace may be facilitated by an expecially suitable choice of these functions.

The subspace $\mathscr{V}_{\mathrm{BpA}}$ is spanned by the vectors

$$
y_{j}^{\prime}=\left(1-\Pi_{A}\right) y_{j}
$$

and it is easily shown that

$$
\Pi_{A} y_{j}=h_{j}\left(\vec{r}_{0}\right) \phi_{o}\left(\vec{\xi}_{1}, \ldots ., \vec{\xi}_{A}\right)
$$

where

$$
\begin{gathered}
h_{j}\left(\vec{r}_{0}\right)=\left(g\left(\vec{r}_{0}, \vec{\xi}_{1}\right) \mid \bar{Y}_{j}\left(\vec{r}_{0}, \vec{\xi}_{1}\right) \bar{\psi}_{D}\left(\vec{r}_{0}, \vec{\xi}_{1}\right)\right)_{\xi(1)} \\
\vec{Y}_{j}\left(\vec{r}_{0}, \vec{\xi}_{1}\right) \equiv Y_{j}\left(\vec{r}_{D}\right)
\end{gathered}
$$

and

$$
\vec{\psi}_{\mathrm{D}}\left(\overrightarrow{\mathbf{r}}_{0}, \vec{\xi}_{1}\right) \equiv \psi_{\mathrm{D}}(\vec{\eta})
$$

Directly from (C8a) and the bound state nature of $\bar{\psi}_{\mathrm{D}}$ follows the property

$$
\mathrm{h}_{\mathrm{j}}\left(\overrightarrow{\mathrm{r}}_{0}\right)-0 \quad \text { when } \quad\left|\overrightarrow{\mathrm{r}}_{0}\right| \rightarrow \infty
$$

which, in turn, implies that $h_{j}$ vanishes at asymptotic separations in channel $\beta$. Also,

$$
\left\langle\mathrm{y}_{\mathbf{j}}^{\dagger} \mid \mathrm{y}_{\mathrm{i}}^{\prime}\right\rangle=\delta_{\mathrm{ij}}-\left(\mathrm{h}_{\mathbf{j}} \mid \mathrm{h}_{\mathbf{i}}\right)
$$

shows that the $\mathrm{y}_{j}^{\prime}$ are not orthogonal or necessarily independent. 
A somewhat different projection space from $\mathscr{V}_{B}$ was employed by Feshbach (ref. 2) in treating the pickup process to illustrate his formalism. This space (hereinafter called $\left.\mathscr{V}_{\mathrm{FB}}\right)$ can be viewed as the larger space obtained by taking the vector sum of subspaces like $\mathscr{V}_{\mathrm{B}}$ over the full set of states $\psi_{i}(\vec{\eta})$, both bound and continuum, of the system $\mathrm{D}$. Then $\mathscr{V}_{\mathrm{FB}}$ correspoilds to all states having the form $\mathrm{z}\left(\overrightarrow{\mathrm{r}}_{\mathrm{D}}, \vec{\eta}\right) \psi_{\mathrm{o}}$, and an orthonormal basis for the space can be expressed as the totality of functions

$$
\mathrm{z}_{\mathrm{mn}}=\mathrm{z}_{\mathrm{mn}}\left(\overrightarrow{\mathrm{r}}_{\mathrm{D}}, \vec{\eta}\right) \psi_{\mathrm{o}}
$$

where the $\mathrm{Z}_{\mathrm{mn}}$ constitute a complete set of functions of $\overrightarrow{\mathrm{r}}_{\mathrm{D}}$ and $\vec{\eta}$ (or $\overrightarrow{\mathrm{r}}_{0}$ and $\vec{\xi}_{1}$ ). A particular choice would be $\mathrm{Z}_{\mathrm{mn}}=\mathrm{Y}_{\mathrm{m}}\left(\overrightarrow{\mathrm{r}}_{\mathrm{D}}\right) \psi_{\mathrm{n}}(\vec{\eta})$. It is assumed that the $\mathrm{Z}_{\mathrm{mn}}$ are normalized so that the closure relation has the form

$$
\sum_{\mathrm{m}, \mathrm{n}} \overline{\mathrm{z}}_{\mathrm{mn}}\left(\overrightarrow{\mathrm{r}}_{0}, \vec{\xi}_{1}\right) \overline{\mathrm{z}}_{\mathrm{mn}}^{*}\left(\overrightarrow{\mathrm{r}}_{0}^{\prime}, \vec{\xi}_{1}^{\prime}\right)=\delta\left(\overrightarrow{\mathrm{r}}_{0}-\overrightarrow{\mathrm{r}}_{0}^{\prime}\right) \delta\left(\vec{\xi}_{1}-\vec{\xi}_{1}^{\prime}\right)
$$

Clearly, the space $\mathscr{V}_{\text {FB }}$ contains the entire component of $\Psi$ on the vector $\psi_{0}$. In addition to the open $\beta$ channel, this space includes the open three-particle channel ( $n, 1$, $\mathscr{A}-1)$ in which the nucleus $\mathscr{A}-1$ is in the state $\psi_{0}$ and, also, the contribution to the channel $\alpha \mathrm{m}$ arising from the component of $\varphi_{\alpha \mathrm{m}}$ on $\psi_{\mathrm{o}}$. Thus, $\mathscr{V}_{\mathrm{FB}}$ can be used for a wider range of conditions than the two-channel case consider ed here. The projector into $\mathscr{V}_{\mathrm{FB}}$ is

$$
\Pi_{\mathrm{FB}}=\sum_{\mathrm{m}, \mathrm{n}}\left|\mathrm{z}_{\mathrm{mn}}\right\rangle\left\langle\mathrm{z}_{\mathrm{mn}}\left|={ }^{\vec{r}_{0}}{ }_{\vec{\xi}_{1}}\right| \psi_{\mathrm{o}}\right\rangle\left\langle\psi_{\mathrm{o}}\right|
$$

The projector that selects the open channels can be taken to be

$$
\begin{aligned}
P_{F} & =\Pi_{F B}+\Pi_{A p F B} \\
& =\Pi_{A}+\Pi_{F B p A}
\end{aligned}
$$

The space $\mathscr{V}_{\mathrm{ApFB}}$ is spanned by the vectors

$$
\begin{aligned}
\mathbf{x}_{\mathbf{i}}^{\prime \prime} & =\mathbf{N}\left(1-\Pi_{\mathrm{FB}}\right) \mathbf{x}_{\mathbf{i}} \\
& =\operatorname{NX}_{\mathbf{i}}\left(\vec{r}_{0}\right)\left[\phi_{0}\left(\vec{\xi}_{1}, \ldots, \vec{\xi}_{\mathrm{A}}\right)-\mathrm{g}\left(\overrightarrow{\mathrm{r}}_{0}, \vec{\xi}_{1}\right) \psi_{\mathrm{o}}\left(\vec{\zeta}_{2}, \ldots, \vec{\zeta}_{\mathrm{A}}\right)\right]
\end{aligned}
$$


whose scalar products are

$$
\mathrm{N}^{-2}\left\langle\mathrm{x}_{j}^{\prime \prime} \mid \mathrm{x}_{i}^{\prime \prime}\right\rangle=\delta_{i j}-\left(\mathrm{x}_{\mathrm{j}}\left(\overrightarrow{\mathrm{r}}_{0}\right) \mathrm{g}\left(\overrightarrow{\mathrm{r}}_{0}, \vec{\xi}_{1}\right) \mid \mathrm{x}_{\mathrm{i}}\left(\overrightarrow{\mathrm{r}}_{0}\right) \mathrm{g}\left(\overrightarrow{\mathrm{r}}_{0}, \vec{\xi}_{1}\right)\right)
$$

Although the $\mathbf{x}_{\mathbf{i}}^{\prime \prime}$ are not, in general, orthogonal to one another, they will be orthogonal if the function $\mathrm{g}$ happens to be independent of $\vec{r}_{0}$. Then the scalar product on the right of equation (C12) will be proportional to the Kronecker delta by virtue of the orthogonality of the functions $\mathrm{X}_{\mathrm{i}}$. In the limit that the nucleus $\mathscr{A}-1$ is infinitely massive compared with $\mathrm{D}$,

$$
\begin{aligned}
\vec{\xi}_{\mathrm{i}} & =\vec{\zeta}_{\mathrm{i}}=\overrightarrow{\mathrm{r}}_{\mathrm{i}} \\
\mathrm{g} & =\mathrm{g}\left(\overrightarrow{\mathrm{r}}_{1}\right) \quad \mathrm{M}_{\mathrm{A}-1} \gg \mathrm{M}_{\mathrm{D}}
\end{aligned}
$$

and the $x_{i}^{\prime \prime}$ constitute an orthogonal set. A function $\phi_{0}^{\prime}$ can then be defined by

$$
\phi_{0}^{\prime}\left(\vec{r}_{1}, \ldots, \vec{r}_{A}\right)=N\left[\phi_{0}-g\left(\vec{r}_{1}\right) \psi_{0}\right]
$$

and (C11a) becomes

$$
\mathrm{x}_{\mathrm{i}}^{\prime \prime}=\mathrm{x}_{\mathrm{i}}\left(\overrightarrow{\mathrm{r}}_{0}\right) \phi_{\mathrm{o}}^{\prime}
$$

The normalization constant $\mathrm{N}$, which makes $\left(\phi_{0}^{\prime} \mid \phi_{0}^{\prime}\right)$ equal to 1 , satisfies the relation

$$
|\mathrm{N}|^{-2}=1-(\mathrm{g} \mid \mathrm{g})
$$

Under these conditions, the explicit expressions for the projection operators in equation (C10a), are as follows:

$$
\begin{aligned}
\Pi_{\mathrm{ApFB}} & =\sum_{\mathbf{i}}\left|\mathrm{x}_{\mathrm{i}}^{\prime \prime}\right\rangle\left\langle\mathrm{x}_{\mathrm{i}}^{\prime \prime}\left|=1_{\overrightarrow{\mathrm{r}}_{0}}\right| \phi_{0}^{\prime}\right\rangle\left\langle\phi_{0}^{\prime}\right| \\
\mathbf{P}_{\mathrm{F}} & ={ }_{\mathbf{r}_{0}}{ }_{\vec{r}_{1}}\left|\psi_{0}\right\rangle\left\langle\psi_{0}\left|+{ }_{\vec{r}_{0}}\right| \phi_{0}^{\prime}\right\rangle\left\langle\phi_{0}^{\prime} \mid \mathbf{M}_{\mathrm{A}-1}\right\rangle \mathbf{M}_{\mathbf{D}}
\end{aligned}
$$

The subspace $\mathscr{A}_{\text {FBpA }}$ is spanned by the vectors 


$$
\begin{aligned}
z_{m n}^{\prime \prime} & =M_{m n}\left(1-\Pi_{A}\right) z_{m n} \\
& =M_{m n}\left[z_{m n}-b_{m n}\left(\vec{r}_{0}\right) \phi_{0}\right]
\end{aligned}
$$

with

$$
\mathrm{b}_{\mathrm{mn}}\left(\overrightarrow{\mathrm{r}}_{0}\right)=\left(\mathrm{g} \mid \mathrm{Z}_{\mathrm{mn}}\right)_{\xi(1)}
$$

Their scalar products are given by the equation

$$
\left(M_{m n^{\prime}} M_{m^{\prime} n^{\prime}}\right)^{-1}\left\langle z_{m n}^{\prime \prime} \mid z_{m^{\prime \prime} n^{\prime}}^{\prime \prime}\right\rangle=\delta_{m m^{\prime}} \delta_{n n^{\prime}}-\left(b_{m n}\left(\vec{r}_{0}\right) \mid b_{m^{\prime} n^{\prime}}\left(\vec{r}_{0}\right)\right)
$$

If the basis set $\mathrm{z}_{\mathrm{mn}}$ is chosen to be

$$
\mathrm{z}_{\mathrm{mn}}=\mathrm{x}_{\mathrm{m}}\left(\overrightarrow{\mathrm{r}}_{0}\right) \lambda_{\mathrm{n}}\left(\vec{\xi}_{1}\right)
$$

then

$$
\mathrm{b}_{\mathrm{mn}}\left(\overrightarrow{\mathrm{r}}_{0}\right)=\mathrm{x}_{\mathrm{m}}\left(\overrightarrow{\mathrm{r}}_{0}\right) \mathrm{c}_{\mathrm{n}}\left(\overrightarrow{\mathrm{r}}_{0}\right)
$$

where

$$
c_{n}\left(\vec{r}_{0}\right)=\left(g\left(\vec{r}_{0}, \vec{\xi}_{1}\right) \mid \lambda_{n}\left(\vec{\xi}_{1}\right)\right)_{\xi(1)}
$$

and the $\lambda_{n}$ are an orthonormal set in the variable $\vec{\xi}_{1}$. If the $c_{n}\left(\vec{r}_{0}\right)$ were independent of $\vec{r}_{0}$ (i.e., if they were constants), it would then follow from the orthogonality of the $\mathrm{x}_{\mathrm{m}}\left(\overrightarrow{\mathrm{r}}_{0}\right)$ that the $\mathrm{b}_{\mathrm{mn}}\left(\overrightarrow{\mathrm{r}}_{0}\right)$ were orthogonal for different values of $\mathrm{m}$. Again, in the limit of an infinitely massive target for which equations (C13a) and (C13b) hold, the required conditions are fulfilled and the $c_{n}$ are constants. In this case, the orthonormal set $\lambda_{n}\left(\vec{r}_{1}\right)$ can be chosen so that

$$
\mathrm{g}\left(\overrightarrow{\mathrm{r}}_{1}\right)=\mathrm{c}_{\mathrm{o}} \lambda_{\mathrm{o}}\left(\overrightarrow{\mathrm{r}}_{1}\right)
$$

where the constant $c_{o}=[(\mathrm{g} \mid \mathrm{g})]^{1 / 2}$. Then the orthonormal basis for the subspace $\mathscr{V}_{\text {FBpA }}$ is 


$$
\mathrm{z}_{\mathrm{mn}}^{\prime \prime}=\mathrm{x}_{\mathrm{m}}\left(\overrightarrow{\mathrm{r}}_{0}\right) \psi_{\mathrm{n}}^{\prime}\left(\overrightarrow{\mathrm{r}}_{1}, \ldots, \overrightarrow{\mathrm{r}}_{\mathrm{A}}\right) \quad \mathrm{M}_{\mathrm{A}-1}>\mathrm{M}_{\mathrm{D}}
$$

where

$$
\begin{gathered}
\psi_{\mathrm{o}}^{\prime}=\mathrm{N}\left[\lambda_{\mathrm{o}}\left(\overrightarrow{\mathrm{r}}_{1}\right) \psi_{\mathrm{o}}-\mathrm{c}_{\mathrm{o}} \phi_{\mathrm{o}}\right] \\
\psi_{\mathrm{n}}^{\prime}=\lambda_{\mathrm{n}}\left(\overrightarrow{\mathrm{r}}_{1}\right) \psi_{\mathrm{o}} \quad \mathrm{n}=1,2, \ldots \\
\left(\phi_{\mathrm{o}} \mid \psi_{\mathrm{n}}^{\prime}\right)=0 \quad \mathrm{n}=0,1,2, \ldots
\end{gathered}
$$

The projector into this space is

$$
\begin{aligned}
\Pi_{\mathrm{FBpA}} & =\sum_{\mathrm{m}, \mathrm{n}}\left|z_{\mathrm{mn}}^{\prime \prime}\right\rangle\left\langle z_{\mathrm{mn}}^{\prime \prime}\left|=1_{\vec{r}_{0}} \sum_{\mathrm{n}}\right| \psi_{\mathrm{n}}^{\prime}\right\rangle\left\langle\psi_{\mathrm{n}}^{\prime}\right| \\
& =1_{\vec{r}_{0}}\left[\left|\psi_{\mathrm{o}}^{\prime}\right\rangle\left\langle\psi_{\mathrm{o}}^{\prime}\left|+1_{\vec{r}_{1}}\right| \psi_{\mathrm{o}}\right\rangle\left\langle\psi_{\mathrm{o}}|-| \lambda_{\mathrm{o}} \psi\right\rangle\left\langle\lambda_{\mathrm{o}} \psi_{\mathrm{o}}\right|\right]
\end{aligned}
$$

In the last step, use was made of the completeness of the set $\lambda_{n}\left(\vec{r}_{1}\right)$. From equations (C7), (C9), and (C15),

$$
\left|\lambda_{0} \psi_{0}\right\rangle=\mathbf{c}_{0}^{-1} \Pi_{F B}\left|\varphi_{0}\right\rangle
$$

can be written. Inserting this form into equation (C16) along with equations (C4) and (C9) gives

$$
\Pi_{F B p A}=|N|^{2}\left(c_{o}^{-1} \Pi_{F B}-c_{o} 1\right) \Pi_{A}\left(c_{o}^{-1} \Pi_{F B}-c_{o} 1\right)+\Pi_{F B}-c_{o}^{-2} \Pi_{F B} \Pi_{A} \Pi_{F B}
$$

After some manipulation, this equation becomes

$$
\Pi_{F B p A}=-\Pi_{A}+\Pi_{F B}+|N|^{2}\left(1-\Pi_{F B}\right) \Pi_{A}\left(1-\Pi_{F B}\right)
$$

or, alternatively,

$$
\Pi_{F B p A}=\left(1-\Pi_{A}\right) \Pi_{F B}+|N|^{2}\left(c_{o}^{2} 1-\Pi_{F B}\right) \Pi_{A}\left(1-\Pi_{F B}\right)
$$


When the form given by equation (C17) is employed in equation (C10b), it immediately gives equation (C10a), as a glance at equations (C14a) and (C11a) will confirm. The form of equation (C18) is the resolution of the operator $\Pi_{F B p A}$ into a part operating on $\mathscr{V}_{\text {FB }}$ and a part operating on the subspace orthogonal to it. Equation (C18) illustrates the relation given by equation (29). 


\section{REFERENCES}

1. Feshbach, Herman: Unified Theory of Nuclear Reactions. Ann. Phys. (NY), vol. 5, no. 4, Dec. 1958, pp. 357-390.

2. Feshbach, Herman: A Unified Theory of Nuclear Reactions. II. Ann. Phys. (NY), vol. 19, no. 2, Aug. 1962, pp. 287-313.

3. Coester, F.; and Kümmel, H.: Time Dependent Theory of Scattering of Nucleons by Nuclei. Nucl. Phys., vol. 9, 1958, pp. 225-236.

4. Mittleman, Marvin H. ; and Watson, Kenneth M. : Scattering of Charged Particles by Neutral Atoms. Phys. Rev., vol. 113, no. 1, Jan. 1, 1959, pp. 198-211.

5. Glauber, R. J.: High-Energy Collision Theory. Lectures in Theoretical Physics. Vol. I. Wesley E. Brittin and Lita G. Dunham, eds., Interscience Publ., Inc., 1959 , pp. 315-414.

6. Kerman, A. K.; McManus, H.; and Thaler, R. M. : The Scattering of Fast Nucleons from Nuclei. Ann. Phys. (N. Y.), vol. 8, no. 4, Dec. 1959, pp. 551-635.

7. Fetter, Alexander L.; and Watson, Kenneth M. : The Optical Model. Advances in Theoretical Physics. Vol. 1, Keith A. Brueckner, ed., Academic Press, 1965, pp. 115-194.

8. Mittleman, H. H. ; and Pu, Robert: Optical Potential for Closely Coupled States. Phys. Rev., vol. 126, no. 1, April 1, 1962, pp. 370-372.

9. Mittleman, Marvin H. : Elastic Scattering of Electrons by Atoms. Advances in Theoretical Physics. Vol. 1, Keith A. Brueckner, ed., Academic Press, 1965, pp. 283-315.

10. Chase, D. M. ; Wilets, L.; and Edmonds, A. R. : Rotational-Optical Model for Scattering of Neutrons. Phys. Rev., vol. 110, no. 5, June 1, 1958, pp. 1080-1092.

11. Buck, B. : Calculation of Elastic and Inelastic Proton Scattering with a Generalized Optical Model. Phys. Rev., vol. 130, no. 2, April 15, 1963, pp. 712-726.

12. Tamura, Taro: Analyses of the Scattering of Nuclear Particles by Collective Nuclei in Terms of the Coupled-Channel Calculation. Rev. Mod. Phys. vol. 37, no. 4, Oct. 1965, pp. 679-708.

13. Pu, Robert Ta-Pang: A Generalized Optical-Potential Method and its Application to the Scattering of Electrons by Atomic Hydrogen. Rep. No. UCRL-10878, University of California, Lawrence Radiation Lab. , June 18, 1963. 
14. Kerman, Arthur K. ; Rodberg, Leonard S.; and Young, James E. : Intermediate Structure in the Energy Dependence of Nuclear Cross Sections. Phys. Rev. Letters, vol. 11 , no. 9 , Nov. 1,1963 , pp. $422-425$.

15. Hahn, Yukap: Projection Operators in the Unified Reaction Theory. Phys. Rev., vol. 142, no. 3, Feb. 18, 1966, pp. 603-607. 


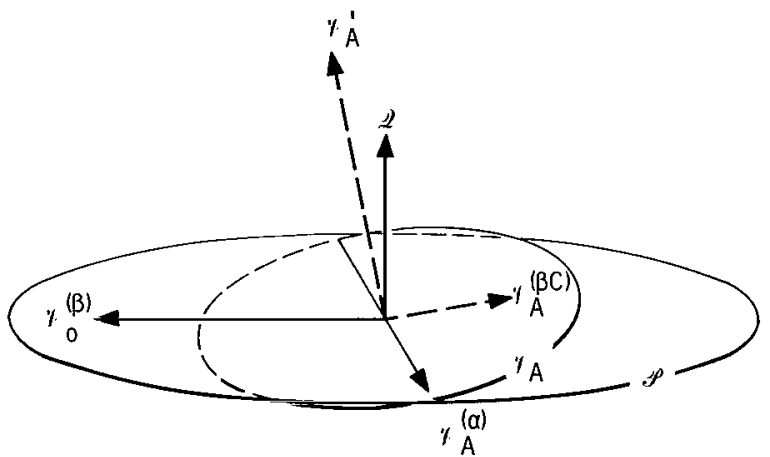

Figure 1. - Schematic representation of projective subspaces $p$ and ${ }^{\prime} \mathrm{A}$. 
"The derondutical and space activities of the United States shall be conducted so as to contribute . . to the expansion of human knowledge of phenomena in the atmosphere and space. The Administration shall provide for the widest practicable and appropriate dissemination of information concerning its activities and the results thereof."

-National Aeronautics and Space ACt of 1958

\section{NASA SCIENTIFIC AND TECHNICAL PUBLICATIONS}

TECHNICAL REPORTS: Scientific and technical information considered important, complete, and a lasting contribution to existing knowledge.

TECHNICAL NOTES: Information less broad in scope but nevertheless of importance as a contribution to existing knowledge.

TECHNICAL MEMORANDUMS: Information receiving limited distribution because of preliminary data, security classification, or other reasons.

CONTRACTOR REPORTS: Scientific and technical information generated under a NASA contract or grant and considered an important contribution to existing knowledge.

TECHNICAL TRANSLATIONS: Information published in a foreign language considered to merit NASA distribution in English.

SPECIAL PUBLICATIONS: Information derived from or of value to NASA activities. Publications include conference proceedings, monographs, data compilations, handbooks, sourcebooks, and special bibliographies.

TECHNOLOGY UTILIZATION PUBLICATIONS: Information on technology used by NASA that may be of particular interest in commercial and other non-aerospace applications. Publications include Tech Briefs, Technology Utilization Reports and Notes, and Technology Surveys.

Details on the availability of these publications may be obtained from:

SCIENTIFIC AND TECHNICAL INFORMATION DIVISION

NATIONAL AERONAUTICS AND SPACE ADMINISTRATION

Washington, D.C. 20546 